\title{
Does feature similarity facilitate attentional selection?
}

\author{
Yariv Festman AND Jochen Braun \\ Otto von Guericke University, Magdeburg, Germany
}

\begin{abstract}
Object-based attention enables us to simultaneously select and report two features from the same visual object. Does feature-based attention contribute similarly to visual selection? In the present study, we investigated the concurrent discrimination of two motion fields with a divided attention paradigm. We found that dual-task performance improved when the two fields conformed to a continuous optic flow, consistent with "object-based" selection. However, we found no such improvement when the two motion fields were merely similar, as would have been expected from "feature-based" selection. Therefore, feature similarity does not facilitate attentional selection in the same way as belonging to the same object does.
\end{abstract}

When observers view two visual targets simultaneously, they are often unable to report attributes of both targets, even though they can readily report attributes of either target alone. In such situations, observers can typically raise the report accuracy for one target only by lowering report accuracy for the other. These canonical observations define the "limited resource" of selective visual attention (Norman \& Bobrow, 1975; Pashler, 1999; Pastukhov, Fischer, \& Braun, 2009; Sperling \& Dosher, 1986).

The difficulty posed by multiple visual targets is not of fixed magnitude, but is modulated by the perceptual organization of a visual scene. When two targets are segmented into the same perceptual unit, their attributes can typically be reported concurrently (Blaser, Pylyshyn, \& Holcombe, 2000; Duncan, 1984; Rodríguez, Valdés-Sosa, \& Freiwald, 2002). Thus, visual attention seems to be able to select - all in one go - multiple attributes associated with a given perceptual unit (or "visual object"). This "objectbased" nature of attentional selection has been confirmed by numerous behavioral studies (integrated competition hypothesis; Driver \& Frith, 2000; Duncan, 1996; Scholl, 2001; Treisman \& Kanwisher, 1998).

Object-specific effects of attention have also been encountered in single-unit activity of visual cortex (Roelfsema, Lamme, \& Spekreijse, 1998), in visual evoked potentials (Schoenfeld et al., 2003; Valdés-Sosa, Bobes, Rodríguez, \& Pinilla, 1998), and in functional imaging signals (O’Craven, Downing, \& Kanwisher, 1999). For example, attending to a task-relevant motion attribute enhances the evoked response to a task-irrelevant color attribute, provided that both are attributes of the same visual object (Schoenfeld et al., 2003).

In recent years, it has become evident that the attention modulation of neural responses also follows a different, "feature-based" principle. Several studies of single-unit activity in visual cortex (Martínez-Trujillo \& Treue, 2004; Maunsell \& Treue, 2006; Treue \& Martínez-Trujillo, 1999; Treue \& Maunsell, 1996), of visual evoked potentials (Andersen, Hillyard, \& Müller, 2008; Müller et al., 2006), and of functional brain imaging (Liu, Larsson, \& Carrasco, 2007; Sàenz, Buracas, \& Boynton, 2002; Serences \& Boynton, 2007) have demonstrated that attending to a particular visual feature elevates neural responses to that same feature not only in the attended region but also throughout the visual field. For example, when an observer attends to upward motion in the left hemifield, neural responses are elevated also to task-irrelevant stimuli in the right hemifield, provided these stimuli exhibit upward motion, too (Sàenz et al., 2002; Treue \& Maunsell, 1996).

However, it is unclear whether this feature-based modulation confers all of the perceptual benefits that are usually associated with attention. In particular, the evidence that stimuli with similar features can be jointly selected by feature-based attention remains unconvincing. On the one hand, Sàenz, Buracas, and Boynton (2003) reported that independent attributes of two target arrays are discriminated more readily when both arrays share a common feature. On the other hand, three separate dual-task studies by our group failed to confirm that shared features, per se, facilitate perception in the sense that attributes of distinct targets become easier to report (Lee, Koch, \& Braun, 1999a, 1999b; Pastukhov et al., 2009).

By far, the strongest perceptual evidence for featurebased attention comes from visual search tasks. When attention can be guided on the basis of a simple feature to a search target that appears amidst distractors, search performance is markedly improved (Müller, Heller, \& Ziegler, 1995; Treisman, 1988; Wolfe, 1994). Interestingly, the full

J.Braun, jochen.braun@ovgu.de 
extent of the improvement is obtained only when the guiding feature(s) remain(s) the same from trial to trial (Wolfe, Horowitz, Kenner, Hyle, \& Vasan, 2004). In other words, the perceptual benefit of feature-based attention is most pronounced when a selectional bias can build up gradually over successive trials (consistent mapping; Schneider \& Shiffrin, 1977; Shiffrin \& Schneider, 1977).

We wished to establish whether feature-based selection is as helpful in dual-task situations as object-based selection is known to be. To this end, we devised dual-task situations in which two distinct parts of the display in some trials shared features (potentially allowing feature-based selection) and in other trials permitted joint segmentation (object-based selection). As is typical for dual-task experiments on object-based selection (Blaser et al., 2000; Duncan, 1984; Rodríguez et al., 2002), we changed the task-relevant features from trial to trial. Due to this "variable mapping" (Schneider \& Shiffrin, 1977; Shiffrin \& Schneider, 1977), any selectional bias in favor of particular features or objects had to form anew for each trial. Note that the maximal benefit of object-based selection (i.e., elimination of dual-task costs) is obtained even when the target object changes from trial to trial (Bauer \& Braun, 2000).

In the present study, observers were required to independently report attributes of two spatially separate fields of moving dots. To manipulate joint segmentation, the two fields either did or did not conform to a continuous optic flow (Figure 1). We reasoned that conformance to a continuous flow would facilitate selection by object-based attention, whereas failure to conform to any continuous flow would likely discourage such selection. Furthermore, we assigned to the two fields either similar or dissimilar motion flows, expecting to thereby either facilitate or discourage selection by feature-based attention. Crucially, this design allowed us to independently manipulate the continuity and similarity of the two motion fields. Thus, we could form configurations that were discontinuous and similar (Figure 1A), continuous and similar (Figure 1B), discontinuous and dissimilar (Figure 1C), or continuous and dissimilar (Figure 1D). Our results showed unambiguously that only continuity, not similarity, facilitated joint selection by attentional mechanisms. On this basis, we conclude that object-based and feature-based mechanisms do not contribute equally to attentional selection.

\section{METHOD}

\section{Subjects}

Four observers (22-35 years of age) participated in the experiment. Apart from the first author, who also participated in the study, observers were naive regarding the purpose of the experiment. All of the observers completed about 30 to $50 \mathrm{~h}$ of training and testing over a period of 2 to 4 months.
A

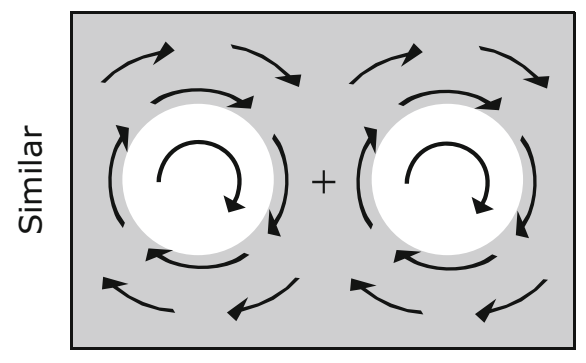

C

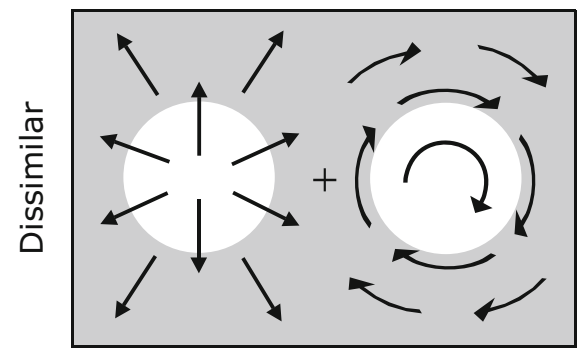

B Continuous

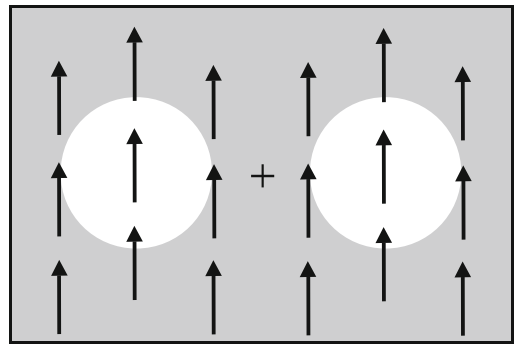

D

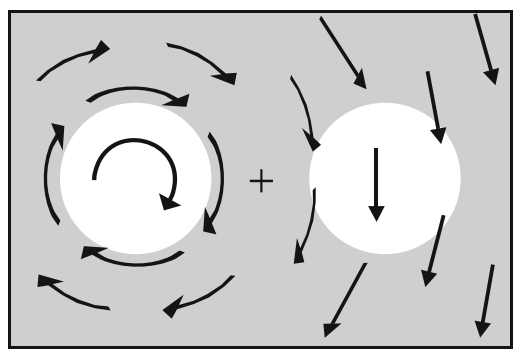

Figure 1. Independent manipulation of similarity and continuity (schematic). Two motion patterns were shown in circular apertures (white regions) to the left and to the right of a fixation cross. The two patterns were either similar $(A, B)$ or dissimilar $(C, D)$, to favor or hinder joint selection by feature-based attention. Independently, the two patterns either did (B, D) or did not $(A, C)$ conform to a continuous overall optic flow, to favor or hinder joint selection by object-based attention. To illustrate the continuity or discontinuity of motion along the vertical midline, each motion pattern above extends throughout its hemifield. In the actual displays, motion patterns were shown only within the circular apertures. (A) Two clockwise circular flows (discontinuous and similar). (B) Two upward flows (continuous and similar). (C) Outward flow and clockwise flow (discontinuous and dissimilar). (D) Clockwise flow and downward flow (continuous and dissimilar). 

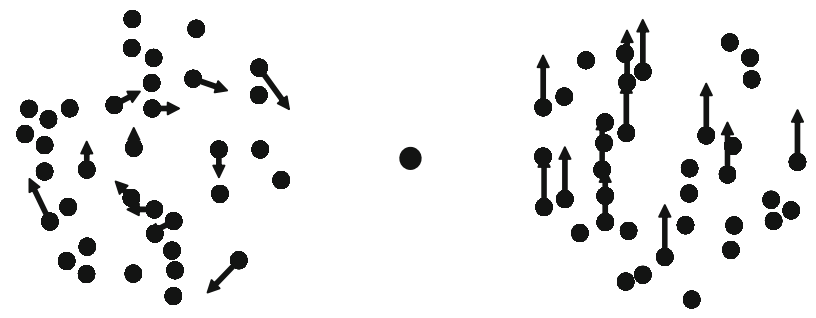

Figure 2. Example visual display (to scale). Two fields of stochastic dot motion were presented within circular apertures $\left(2.5^{\circ}\right)$, centered symmetrically $6.4^{\circ}$ to the left and to the right of a small fixation dot (10' diameter). Each aperture contained 100 dots $\left(6^{\prime}\right.$ diameter). Motion coherence was defined as the proportion of dots chosen anew on each frame that followed a particular flow pattern, illustrated here by arrows extending from coherent dots (clockwise flow in the left aperture and upward flow in the right aperture). The remaining dots were replotted randomly on each frame (frame rate $100 \mathrm{~Hz}$ ).

\section{Apparatus and Stimuli}

Observers were seated at a viewing distance of $85 \mathrm{~cm}$ from a 22in. color monitor (Iiyama; $26^{\circ} \times 21^{\circ}$ usable field of view, $100 \mathrm{~Hz}$ ) and responded in each trial via a standard keyboard. The stimuli were fields of coherently moving dots, which were generated in MATLAB using the Psychophysics Toolbox (Brainard, 1997; Pelli, 1997). For each trial, two circular fields of dots (radius $2.5^{\circ}$ of visual angle) were presented symmetrically to the left and to the right of a small fixation (centered at $6.4^{\circ}$ eccentricity) that was marked by a small dot. Each field contained 100 dots (diameter $6^{\prime}$ of visual angle). Motion coherence was defined as the proportion of dots that moved in accordance with a given flow pattern after each refresh. The remaining dots were replotted at a random location (Figure 2). Four major flow patterns were used (Figure 3 ), and each could have one of two directions: horizontal flow leftward $(l)$ or rightward $(r)$, vertical flow upward $(u)$ or downward $(d)$, circular flow clockwise $(c w)$ or counterclockwise $(c c)$, and radial flow inward (in) or outward (out).

\section{Procedure}

The observers initiated each trial with a keypress. After a short delay $(50-150 \mathrm{msec})$, the stimuli were presented for $250 \mathrm{msec}$, and subjects produced one or two unspeeded responses. During each block of 80 trials, two types of motion patterns were used, one in the left and one in the right motion field (e.g., a block might present vertical patterns in the left field and circular patterns in the right field). However, the direction of each pattern was chosen randomly and independently on every trial. (In the example, the left motion would be either upward or downward and, independently, the right motion would be either clockwise or counterclockwise.) The observers were instructed to maintain fixation and to discriminate the direction of motion either in the left field ("up" or "down"?), in the right field ("clockwise" or "counterclockwise"?), or in both fields.

The observers' performance was measured in two types of conditions. In a single-task condition, the observers attended only one motion field (e.g., the left field) and ignored the other motion field, but nevertheless maintained fixation. In this condition, observers reported the motion direction of the attended field with one keypress. In the dual-task condition, observers attended to both the left and right fields (i.e., they divided their attention), while maintaining fixation. In this condition, observers reported the motion directions of each field with a separate keypress. The first keypress pertained to the left field.

The observers trained for at least 1,000 trials (i.e., several hours) in the single-task condition. Motion coherence was set initially at $60 \%$ and reduced after each block in which performance exceeded $85 \%$. Training was terminated after a subject's performance had stabilized. This procedure was used to select for each pattern type (vertical, horizontal, circular, or radial) and for a coherence level that allowed approximately $85 \%$ correct performance. Then, for each pair of flow patterns, performance was measured in 10 single-task blocks on the left pattern, 10 single-task blocks on the right pattern, and 10 dual-task blocks on both patterns.

\section{Data Analysis}

Performance dependencies. In the dual-task condition, we monitored variability in the division of attention between the left and right patterns (Lee et al., 1999a). If this division varies trial to trial, one expects the success or failure of one task to be negatively correlated with success or failure of the other. On the other hand, if

A
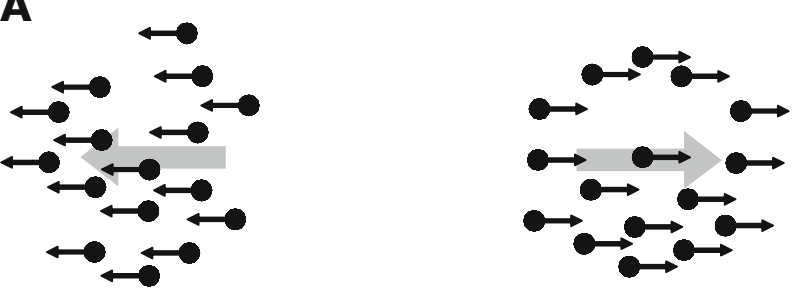

B
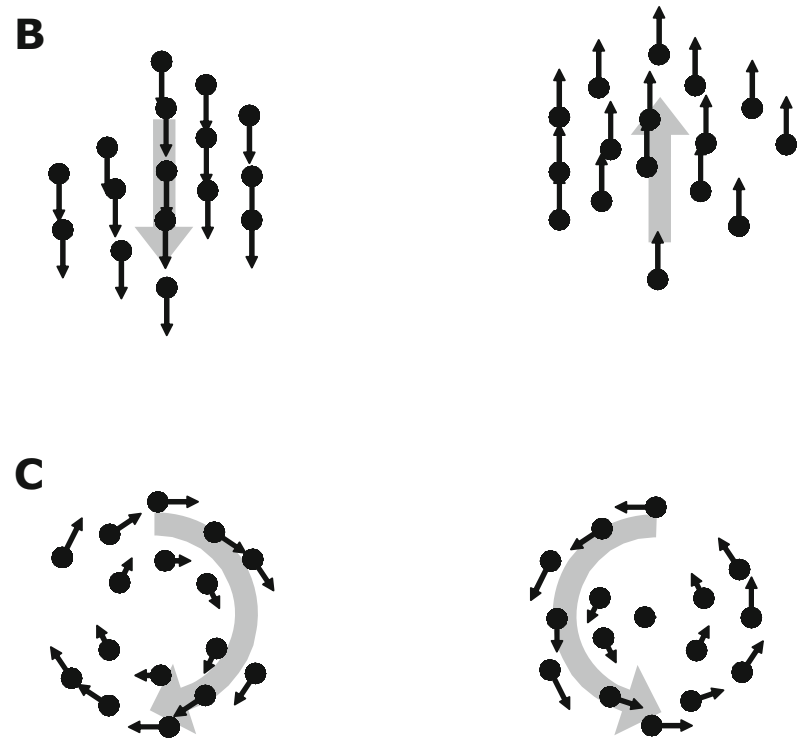

D
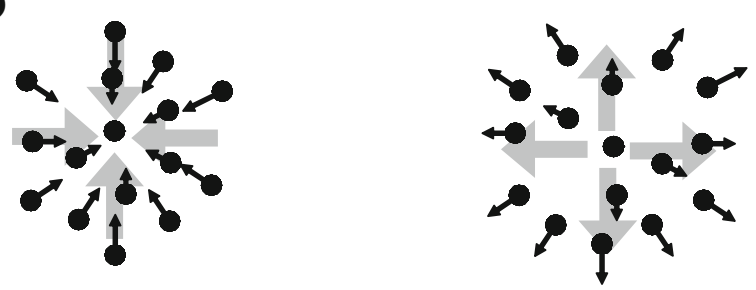

Figure 3. Types of motion fields used (schematic). Thin arrows depict the dot position in the next frame. Thick gray arrows show the overall motion pattern. (A) Horizontal flow leftward or rightward. (B) Vertical flow downward or upward. (C) Circular flow clockwise or counterclockwise. (D) Radial flow inward or outward. 
left and right performance are independent, the actual number $n_{i j}$ of trials with outcome $i$ in the left task and outcome $j$ in the right task should be close to the expected average number $n_{i j}$ of such trials:

$$
\overline{n_{i j}}=\frac{\left(n_{i 0}+n_{i 1}\right)\left(n_{0 j}+n_{1 j}\right)}{\left(n_{00}+n_{11}+n_{01}+n_{10}\right)} .
$$

The hypothesis of independence may be rejected if the $\chi^{2}$ measure of association reaches a sufficiently high value.

$$
\chi^{2}=\sum_{i, j} \frac{\left(n_{i j}-\overline{n_{i j}}\right)^{2}}{\overline{n_{i j}}}
$$

Fourteen percent of the total blocks were found to be significantly correlated (negatively or positively, $p<.05$ ), indicating that the division of attention varied from trial to trial. These blocks were discarded, and further analysis focused on the remaining blocks in which the division of attention remained constant from trial to trial.

Discriminability in the $\boldsymbol{z}$-score plane. We used four types of motion flow: leftward/rightward translation, upward/downward translation, clockwise/counterclockwise rotation, and radial expansion/contraction. To simplify notation, we arbitrarily denoted one direction with "+" and the other with "-" (e.g., "+" for downward and "-" for upward translation). After computing the proportion of correct responses and the $z$-score (separately for "+" and "- " patterns), we computed the discriminability $d^{\prime}$ of these two directions:

$$
\begin{aligned}
& P_{ \pm}^{\text {correct }}=\frac{N_{ \pm}^{\text {correct }}}{N_{ \pm}^{\text {total }}}, \\
& d^{\prime}=z\left\{P_{+}^{\text {correct }}\right\}-z\left\{P_{-}^{\text {correct }}\right\} .
\end{aligned}
$$

This calculation was carried out separately for right and left motion fields.

The results showed that discriminability $d^{\prime}$ of the left field often depended strongly on the motion flow in the right field (and vice versa). This made it necessary to distinguish all four possible flow combinations in both fields, which we denoted " + +," ",-- , ",+- ," and "-+." In this notation, the left and right symbols refer to left and right motion fields, respectively.

Proportions correct, $L$ and $R$, and the corresponding $z$-scores, $l$ and $r$, were calculated for the left and right motion fields, respectively:

$$
\begin{array}{ll}
L_{ \pm \pm}=\frac{N_{ \pm \pm}^{\text {left }}}{N_{ \pm \pm}^{\text {total }}}, & R_{ \pm \pm}=\frac{N_{ \pm \pm}^{\text {right }}}{N_{ \pm \pm}^{\text {total }}} \\
l_{ \pm \pm}=z\left\{L_{ \pm \pm}\right\}, & r_{ \pm \pm}=z\left\{R_{ \pm \pm}\right\} .
\end{array}
$$

For example, $L_{-+}$denotes the proportion of correct responses to a "-" motion in the left field, given a "+" motion in the right field.

To illustrate graphically the interdependence of left and right motion fields, we plot the results in a " $z$-score plane" (Figure $4 \mathrm{~A}$ ), with left-field $z$-scores represented by the abscissa and right-field $z$-scores by the ordinate. Discriminabilities $d^{\prime}$ can then be calculated either on the basis of one response (distance along one axis) or on the basis of both responses (distance in the $z$-score plane). A compact summary of the results is afforded by the discriminabilities of complementary motion pairs, which may be computed from the observed $z$-score values as follows:

$$
\begin{aligned}
& d_{++v s .--}^{\prime}=\sqrt{\left(l_{++}-l_{--}\right)^{2}+\left(r_{++}-r_{--}\right)^{2}}, \\
& d_{+-v s .-+}^{\prime}=\sqrt{\left(l_{+-}-l_{-+}\right)^{2}+\left(r_{+-}-r_{-+}\right)^{2}} .
\end{aligned}
$$

Here, the first value is the discriminability of the motion field combinations "++" and "- - " (e.g., between two downward and two upward motions), and the second value is the discriminability of motion field combinations "+-" and "-+" (e.g., left downward and right upward and the opposite combination).

Comparing single- and dual-task performance. Our analyses of single- and dual-task performance were identical in all respects. In both cases, we converted proportions correct into $z$-scores, which could be visualized in the $z$-score plane. Specifically, we computed the theoretical discriminability of complementary pairs of motion fields, with

$$
d_{++v s .--}^{\text {single }}, \quad d_{+-v s .-+}^{\text {single }}
$$

computed from single-task $z$-score values and

$$
d_{++v s .--}^{\text {dual }}, \quad d_{+-v s .-+}^{\text {dual }}
$$

computed from dual-task $z$-score values, both according to Equation 5 .

In the absence of capacity limitations, we expect identical performance under single- and dual-task conditions. On this assumption, the single-task performance predicts the maximal dual-task discriminabilities as follows:

$$
\begin{aligned}
& d_{++v s .--}^{\prime \max }=d_{++v s .--}^{\prime \text { single }}, \\
& d_{+-v s .-+}^{\prime \max }=d_{+-v s .-+}^{\prime \text { single }} .
\end{aligned}
$$

If capacity limitations force observers to divide attentional resources, we expect an approximately linear trade-off in the respective performance levels (Lee et al., 1999a, 1999b; Pastukhov et al., 2009). At the midpoint of a linear trade-off, performance on either task will be reduced from $P_{\text {correct }}$ to $\left(P_{\text {correct }}+P_{\text {chance }}\right) / 2$. On this assumption, the single-task performance predicts the following minimal dual-task discriminabilities:

$$
\begin{aligned}
& l_{ \pm \pm}^{\min }=z\left\{\frac{L_{ \pm \pm}+P_{\text {chance }}}{2}\right\}, \\
& r_{ \pm \pm}^{\min }=z\left\{\frac{R_{ \pm \pm}+P_{\text {chance }}}{2}\right\}, \\
& d_{++v s .--}^{\prime \min }=\sqrt{\left(l_{++}^{\min }-l_{--}^{\min }\right)^{2}+\left(r_{++}^{\min }-r_{--}^{\min }\right)^{2}}, \\
& d_{+-v s .-+}^{\prime \min }=\sqrt{\left(l_{+-}^{\min }-l_{-+}^{\min }\right)^{2}+\left(r_{+-}^{\min }-r_{-+}^{\min }\right)^{2}},
\end{aligned}
$$

where $P_{\text {chance }}=.5$.

The maximal and minimal expectations for dual-task discriminabilities are illustrated in Figure 4B and correspond, respectively, to the distances between black symbols (triangles and circles) and between gray symbols (triangles and circles) in the $z$-score plane.

To compare the observed dual-task performance with these two extremes, we computed a "compatibility index" C (Figure 4C),

$$
C=\frac{d^{\prime \text { dual }}-d^{\prime \min }}{d^{\prime \max }-d^{\prime \min }}
$$

which ranges from zero to unity. A compatibility value near unity indicates that $d^{\prime}$ dual is close to $d^{\prime}$ max , implying that the left and right tasks do not compete for attentional resources. A value near zero indicates that $d^{\prime}$ dual is close to $d^{\prime}$ min, and that dividing attention imposes the maximal performance cost.

Quantitation of continuity and similarity. To assess the degrees of continuity and similarity of the left and right motion fields, we devised suitable quantitative measures. Generally speaking, we assessed similarity in terms of how well the left and right motion fields matched each other, and continuity in terms of how well the two fields matched a hypothetical continuous optic flow covering the entire display.

Specifically, we computed a similarity index $M_{\text {sim }}$ as the average directional similarity of motion at 100 corresponding points in the left and right fields. This index takes values near zero when directional differences are much above $30^{\circ}$ and values near unity 


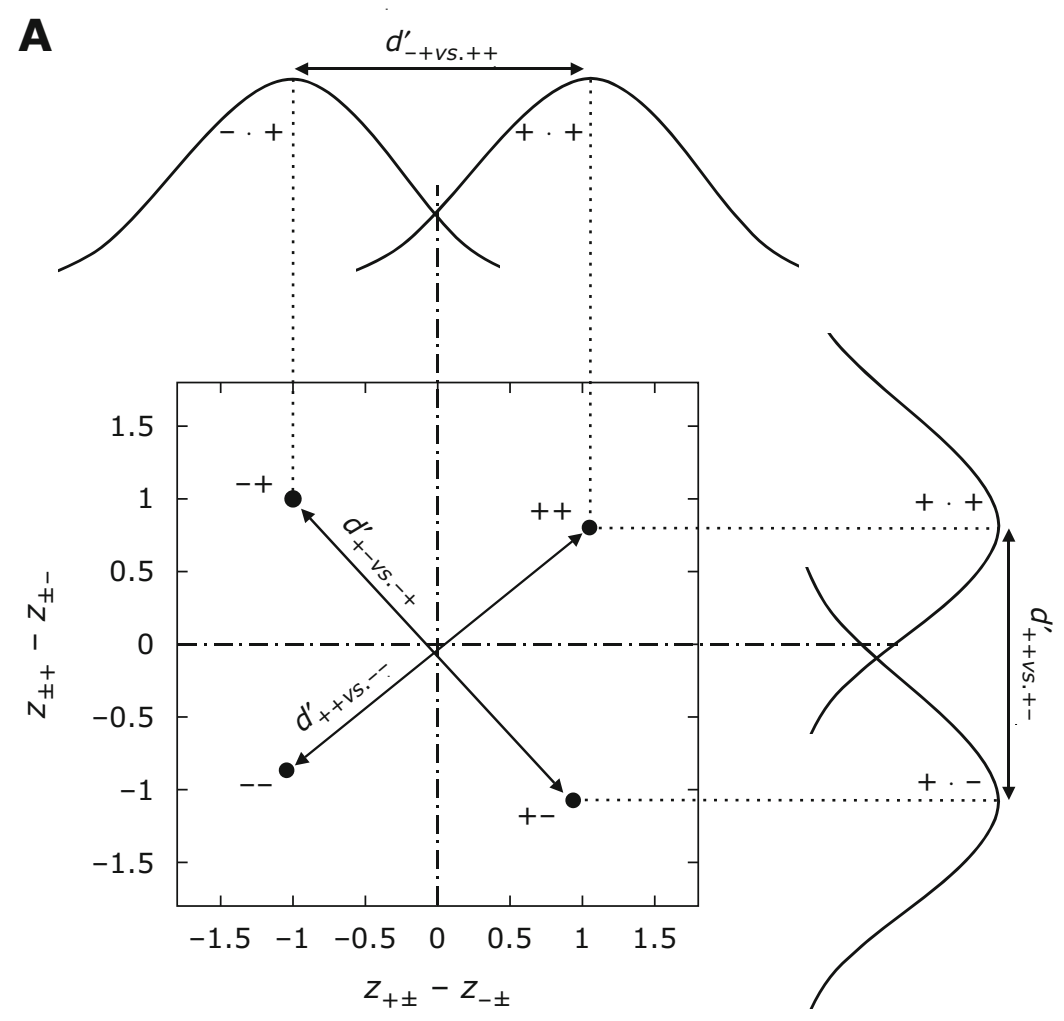

B
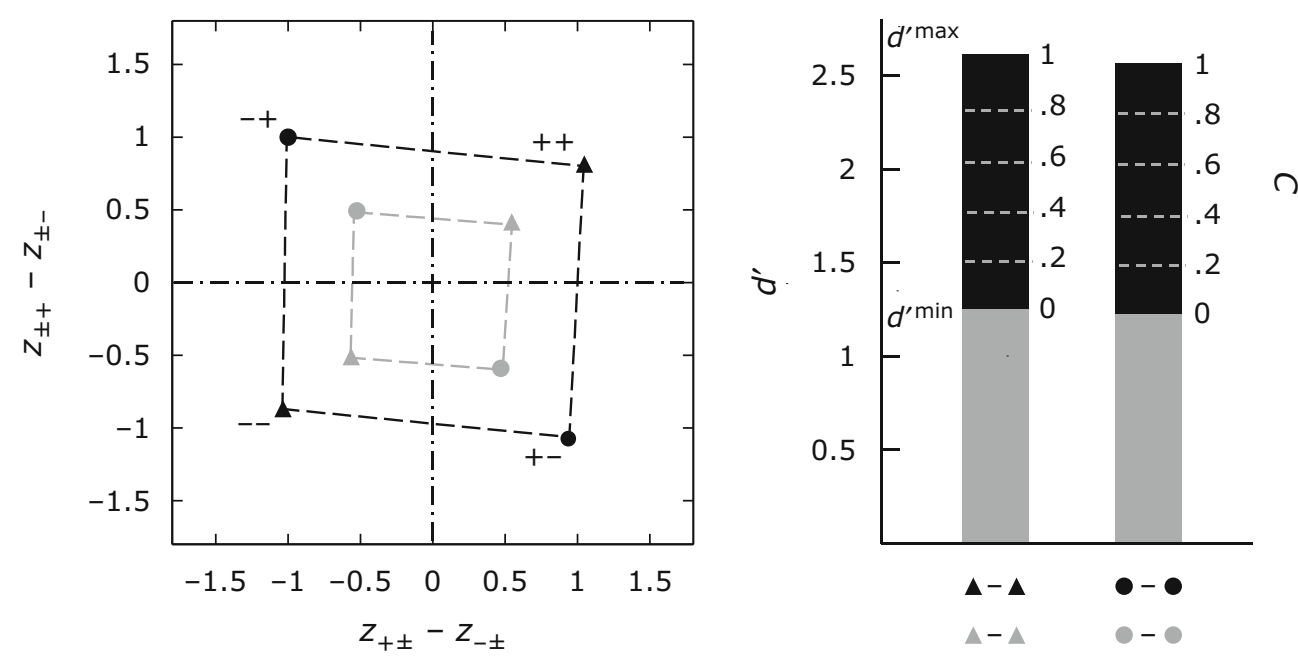

Figure 4. Signal-detection analysis of single- and dual-task performance. (A) Decision signals for discriminating left (abscissa) and right (ordinate) motion fields create a decision space ( $z$-score plane). Hypothetical signal distributions for two stimulus combinations are shown on top ("-+" vs. "++") and on the right ("++" vs. "+-"). Two additional distributions are omitted for clarity ("-+" vs. "- -" on the left, "- -" vs. "+ -" on bottom). Distribution peaks are projected into the decision space along dotted lines; decision criteria are marked by dash-dotted lines. Each stimulus combination has a joint distribution peak in the corresponding quadrant of the space (black circles labeled,,-++++- , and -- ). The discriminability $d^{\prime}$ of two stimulus combinations is the distance between the corresponding peaks, which may be calculated either along one axis (i.e., from the performance of one task) or in the plane (i.e., from the performance of both tasks). A particularly compact summary of the situation is afforded by the discriminabilities of complementary stimulus combinations (diagonal arrows $d_{++s_{-}-t_{-}}^{\prime} d_{+s_{-}++}^{\prime}$ ). (B) Decision space with single-task performance (black triangles and circles) and minimal dual-task performance (gray triangles and circles) halfway between single-task and chance performance (see the Method section). The observed dual-task performance should fall within these extremes. (C) Comparison of dual-task performance with maximal and minimal expectations, on the basis of the discriminabilities of complementary stimulus combinations (diagonal arrows in A): $d_{+-v s .-+}^{\prime}$ is represented by the distances between circles, and $d_{++v s .--}^{\prime}$ by the distances between triangles in the plane. The inset scales represent the compatibility index $C$. Its value is zero when dual-task discriminability matches the minimal expectations $\left(d^{\prime}\right.$ min) and unity when it conforms to the maximal expectations $\left(d^{\prime}\right.$ max $)$. 


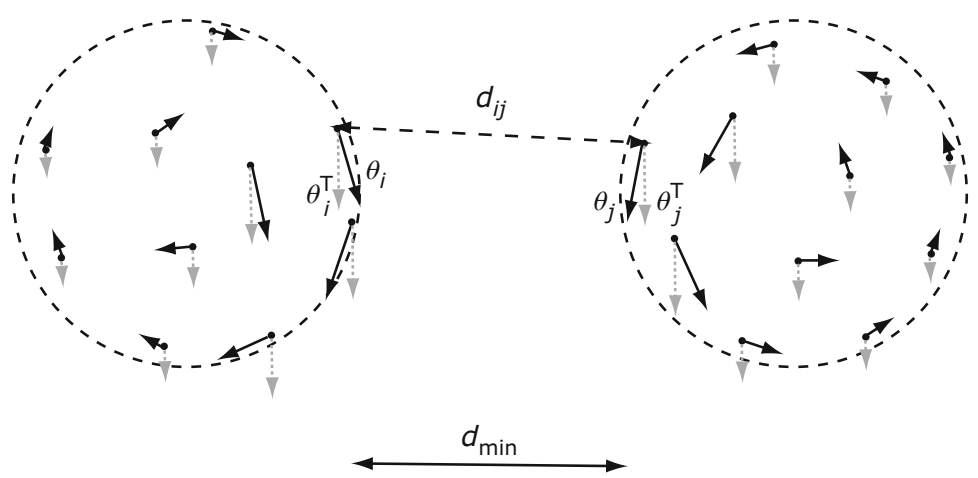

Figure 5. Translational continuity $M_{\mathrm{T}}$ of two counter-rotating motion fields (schematic). Black arrows denote rotational motion in the left (e.g., $\left.\theta_{i}\right)$ and right (e.g., $\theta_{j}$ ) fields. Gray arrows represent a continuous translational optic flow at the same locations (e.g., $\theta_{i}^{\mathrm{T}}, \theta_{j}^{\mathrm{T}}$ ). To obtain $M_{\mathrm{T}}$, the similarity between $\theta$ and $\theta^{\mathrm{T}}$ is computed at all points $i$ and $j$, on a numerical scale from zero to unity (from least to most similar). Next, a weighted average of these similarities is computed over all pairs $i j$, with a weight that increases from the most distant to the most proximal pair, emphasizing the innermost points of the two motion fields. In the present example, the translational continuity is $M_{\mathrm{T}}=.43$, capturing the fact that the innermost points of both fields conform to a continuous downward translation. Pair distance $d_{i j}$; minimal distance $d_{\text {min }}$.

when they are substantially below $30^{\circ}$. The similarity index $M_{\text {sim }}$ is computed as

$$
M_{\text {sim }}=\frac{\sum_{i, j} \exp \left(-\frac{\left(\theta_{i}-\theta_{j}\right)^{2}}{2 \sigma_{\theta}^{2}}\right)}{N},
$$

where the sum goes over $N=100$ randomly chosen corresponding points $i, j$ in the left and right motion fields, respectively, where $\theta_{i}$, $\theta_{j}$ are the local directions of motion at these points, and where $\sigma_{\theta}=$ $30^{\circ}$ is the similarity threshold.

The continuity index $M_{\text {cont }}$ was computed in two steps. First, we computed indices for translational, circular, or radial continuity, termed $M_{\mathrm{T}}, M_{\mathrm{C}}$, and $M_{\mathrm{R}}$, respectively, by matching the two motion fields to continuous optic flows of each type. Second, the final index $M_{\text {cont }}$ was then taken to be the maximal value of the individual indices $M_{\mathrm{T}}, M_{\mathrm{C}}$, and $M_{\mathrm{R}}$ :

$$
M_{\text {cont }}=\max \left(M_{\mathrm{T}}, M_{\mathrm{C}}, M_{\mathrm{R}}\right) .
$$

Accordingly, $M_{\text {cont }}$ measures how well two motion fields conform to the best-matching type of optic flow, whatever type this may be.

The individual indices $M_{\mathrm{T}}, M_{\mathrm{C}}$, and $M_{\mathrm{R}}$ were computed as the average directional similarity of motion (at 200 corresponding points) between the left and right fields, on the one hand, and a hypothetical continuous optic flow, on the other hand. Their values are near zero when the directional differences are much above $30^{\circ}$ and near unity when they are substantially below $30^{\circ}$.

Hypothetical optic flows were specified in terms of a directional sign \pm , the orientation $\theta_{0}$ of a translational flow, and the center coordinates $x_{0}$ and $y_{0}$ of a radial or circular flow. At any given point $x, y$, the local direction of motion was given by

$$
\begin{aligned}
& \theta^{\mathrm{T}}= \pm \theta_{0}, \\
& \theta_{(x, y)}^{\mathrm{C}}= \pm \arctan \frac{x-x_{0}}{y-y_{0}}, \\
& \theta_{(x, y)}^{\mathrm{R}}= \pm \arctan \frac{y-y_{0}}{x-x_{0}},
\end{aligned}
$$

where the sign indicates the direction (upward or downward, inward or outward, clockwise or counterclockwise).
The directional similarity between the motion of points $i$ or $j$ in the left or right motion fields, on the one hand, and the corresponding points in a continuous optic flow, on the other hand, was computed as

$$
\begin{aligned}
& M_{\mathrm{T}, \mathrm{C}, \mathrm{R}}^{i}=\exp \left(-\frac{\left(\theta_{i}-\theta_{i}^{\mathrm{T}, \mathrm{C}, \mathrm{R}}\right)^{2}}{2 \sigma_{\theta}^{2}}\right), \\
& M_{\mathrm{T}, \mathrm{C}, \mathrm{R}}^{j}=\exp \left(-\frac{\left(\theta_{j}-\theta_{j}^{\mathrm{T}, \mathrm{C}, \mathrm{R}}\right)^{2}}{2 \sigma_{\theta}^{2}}\right),
\end{aligned}
$$

where $\theta_{i}$ and $\theta_{j}$ specify the directions at points $i$ and $j$ in the left and right fields, and $\theta_{i}^{\mathrm{T}, \mathrm{C}, \mathrm{R}}$ and $\theta_{j}^{\mathrm{T}, \mathrm{C}, \mathrm{R}}$ specify the directions at corresponding points in the continuous optic flow. Since $\sigma_{\theta}=30^{\circ}$, this results in values near zero when directional differences are much above $30^{\circ}$ and near unity when they are much below $30^{\circ}$.

In combining these values from 200 points (100 each in the left and right fields), we emphasized the most proximal points in the two fields with the help of yet another weighting factor $w_{i j}$,

$$
w_{i j}=\exp \left(-\frac{d_{i j}^{2}}{2 \sigma_{d}^{2}}\right), \quad d_{i j}=\sqrt{\left(x_{i}-x_{j}\right)^{2}-\left(y_{i}-y_{j}\right)^{2}}-d_{\min },
$$

where $\sigma_{d}=1.2^{\circ}$ and $d_{\min }=3.7^{\circ}$ (the smallest distance between the two motion fields).

Specifically, the individual indices $M_{\mathrm{T}}, M_{\mathrm{C}}$, and $M_{\mathrm{R}}$ were then computed. $M_{\mathrm{T}, \mathrm{C}, \mathrm{R}}$ were calculated as

$$
M_{\mathrm{T}, \mathrm{R}, \mathrm{C}}=\max _{\mathrm{T}, \mathrm{R}, \mathrm{C}} \frac{\sum_{i, j} w_{i j} \cdot M_{\mathrm{T}, \mathrm{R}, \mathrm{C}}^{i} \cdot M_{\mathrm{T}, \mathrm{R}, \mathrm{C}}^{j}}{\sum_{i, j} w_{i j}},
$$

where the sum goes over all pairs of points $i, j$, and the maximum is taken over all hypothetical flows of one particular type.

As an example, consider two counter-rotating motion fields (Figure 5). Due to the special weight afforded to the most proximal points of the two fields, the translational continuity of this combination is $M_{\mathrm{T}}=.43$. This captures the fact that the innermost points of the two fields conform well to a continuous downward translation. Two counter-rotating motion fields match even better to circulation 
A
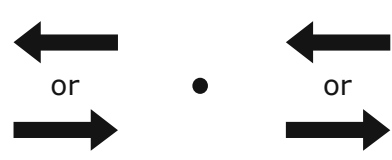

B

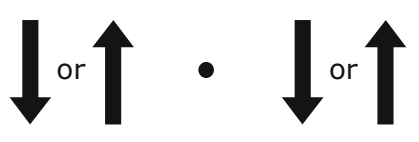

C

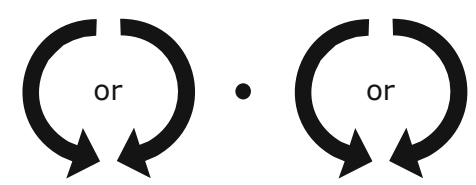

D

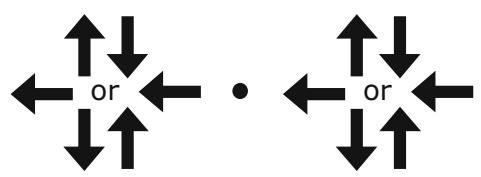

E

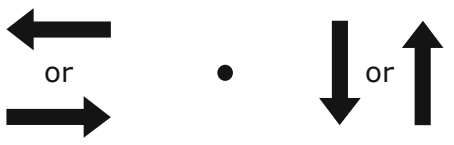

$\mathbf{F}$

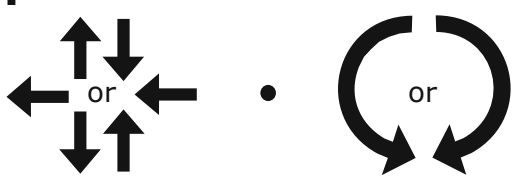

G

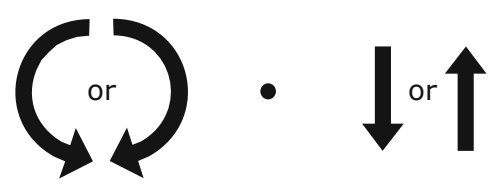

H

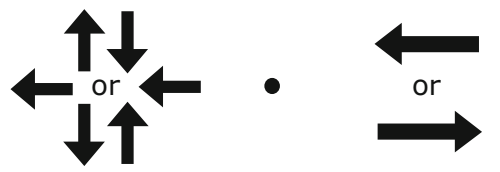

Figure 6. Eight combinations of motion fields were investigated (schematic). Left and right fields contain either similar (A-D) or dissimilar (E-H) motion fields. (A) Two horizontal motions. (B) Two vertical motions. (C) Two circular motions. (D) Two radial motions. (E) Horizontal and vertical motions. (F) Radial and circular motions. (G) Circular and vertical motions. (H) Radial and horizontal motions.

continuity with a center on the left or right field to a value $M_{\mathrm{C}}=$ .63. In contrast, the radial continuity is $M_{\mathrm{R}}=.10$, reflecting the fact that this combination of motion fields matches no radial flow. The overall continuity index is thus $M_{\text {cont }}=M_{\mathrm{C}}=.63$.

\section{RESULTS}

We established discrimination performance for eight combinations of motion fields (Figure 6). Four of these combined similar motion patterns in the left and right fields of view (two horizontal translations, two vertical translations, two circular patterns, and two radial patterns; see Figures 6A-6D). The remaining four combined dissimilar motion patterns (vertical and horizontal translations, rotational and radial patterns, circular motion and vertical translation, radial motion and horizontal translation; see Figures $6 \mathrm{E}-6 \mathrm{H})$. Some of these eight combinations of motion fields conformed better to a continuous optic flow than did other combinations. Table 1 provides quantitative ratings of similarity and continuity for all eight combinations (see the Method section).

\section{Training Results}

All of the observers trained for several hours (at least 1,000 trials) on each of the four display types. During training, motion coherence was reduced gradually until each observer obtained a single-task performance of approximately $85 \%$ correct. Table 2 presents the average coherence and performance levels attained at the end of training. Subjects required substantially less coherence to discriminate circular patterns than to discriminate radial patterns. Similarly, vertical motion required less coher-

Table 1

Quantitative Ratings of Similarity $\left(M_{\text {sim }}\right)$ and Continuity $\left(M_{\text {cont }}\right)$ for All Combinations of Motion Fields

\begin{tabular}{|c|c|c|c|}
\hline Condition & $\begin{array}{l}\text { Motion Field } \\
\text { Combination }\end{array}$ & $M_{\text {sim }}$ & $M_{\text {cont }}$ \\
\hline \multirow[t]{2}{*}{ Horizontal-horizontal } & $1-r, r-1$ & 0 & .39 \\
\hline & $1-1, r-r$ & 1 & 1 \\
\hline \multirow[t]{2}{*}{ Vertical-vertical } & $\mathrm{u}-\mathrm{d}, \mathrm{d}-\mathrm{u}$ & 0 & .71 \\
\hline & $\mathrm{u}-\mathrm{u}, \mathrm{d}-\mathrm{d}$ & 1 & 1 \\
\hline \multirow[t]{2}{*}{ Circular-circular } & $\mathrm{cc}-\mathrm{cW}, \mathrm{cW}-\mathrm{cc}$ & 0 & .63 \\
\hline & $\mathrm{cc}-\mathrm{cc}, \mathrm{cw}-\mathrm{cw}$ & 1 & .20 \\
\hline \multirow[t]{2}{*}{ Radial-radial } & in-out, out-in & 0 & .15 \\
\hline & in-in, out-out & 1 & .04 \\
\hline \multirow[t]{2}{*}{ Horizontal-vertical } & $\mathrm{u}-\mathrm{r}, \mathrm{d}-\mathrm{l}$ & 0 & .08 \\
\hline & $\mathrm{u}-1, \mathrm{~d}-\mathrm{r}$ & 0 & .08 \\
\hline \multirow[t]{2}{*}{ Radial-circular } & in $-\mathrm{cw}$, out $-\mathrm{cc}$ & .08 & .18 \\
\hline & in-cc, out-cw & .08 & .18 \\
\hline \multirow[t]{2}{*}{ Circular-vertical } & $\mathrm{cc}-\mathrm{d}, \mathrm{cw}-\mathrm{u}$ & .25 & .43 \\
\hline & $\mathrm{cc}-\mathrm{u}, \mathrm{cw}-\mathrm{d}$ & .25 & .97 \\
\hline \multirow[t]{2}{*}{ Radial-horizontal } & in $-\mathrm{r}$, out -1 & .23 & .01 \\
\hline & in -1 , out-r & .23 & .53 \\
\hline
\end{tabular}


Table 2

Motion Coherence and Performance Levels (Mean and Standard Error) Obtained From Training the Discrimination of Motion Direction for Four Major Flow Patterns

\begin{tabular}{ccrccc}
\multicolumn{2}{c}{ Motion Direction for Four Major Flow Patterns } \\
\hline $\begin{array}{c}\text { Flow } \\
\text { Pattern }\end{array}$ & \multicolumn{2}{c}{$\begin{array}{c}\text { Coherence } \\
{[\% \text { coherence }]}\end{array}$} & & \multicolumn{2}{c}{$\begin{array}{c}\text { Performance } \\
{[\% \text { correct }]}\end{array}$} \\
\cline { 2 - 5 } & $M$ & $S E$ & & $M$ & $S E$ \\
\hline Horizontal & 26 & 11 & 86.0 & 1.6 \\
Vertical & 15 & 6 & 84.5 & 1.3 \\
Circular & 10 & 4 & 83.3 & 1.3 \\
Radial & 26 & 7 & 86.8 & 1.5 \\
\hline
\end{tabular}

ence than did horizontal motion. The coherence levels determined during training for each subject and motion type were retained for all subsequent experiments.

\section{Test Results}

Subjects performed 10 blocks (a total of 800 trials) for each of three attentional conditions: two single-task conditions (attend left and attend right) and a double-task condition (divide attention between left and right). Task performance was analyzed and summarized in terms of the discriminabilities $d^{\prime}$ of complementary motion field combinations (Figure 4A, diagonal arrows). The dualtask discriminabilities were compared to the maximal and minimal predictions made on the basis of single-task dis- criminabilities (Figure 4B). The resulting "compatibility index" $C$ quantified the performance cost of dividing attention between two motion fields (Figure 4C). See the Method section for details.

We expected that the similarity of two motion fields should facilitate joint selection by feature-based attention, whereas continuity should facilitate joint selection by object-based attention. Accordingly, it would constitute evidence for feature-based attention if dual-task compatibility were to be systematically higher for similar than for dissimilar motion fields. Similarly, we would interpret as evidence for object-based attention if dual-task compatibility proved systematically higher for continuous than for discontinuous motion fields.

Two translational motion fields. We investigated displays with identical and with opposite translational motion in the left and right fields, using either horizontal or vertical motions in both fields. The identical combinations were $1-1, r-r, u-u$, and $d-d$, and the opposite combinations were $1-r, r-1, u-d$, and $d-u$ (Figures $3 \mathrm{~A}$ and $3 \mathrm{~B}$ ). Although identical and opposite motions contrasted starkly in terms of similarity $\left(M_{\mathrm{sim}}=1\right.$ and 0 , respectively), the difference in terms of continuity was less extreme. Due to the fact that opposite vertical motion fields partially conform to a continuous rotational flow with a center at fixation, the continuity indices for identical and opposite vertical
A

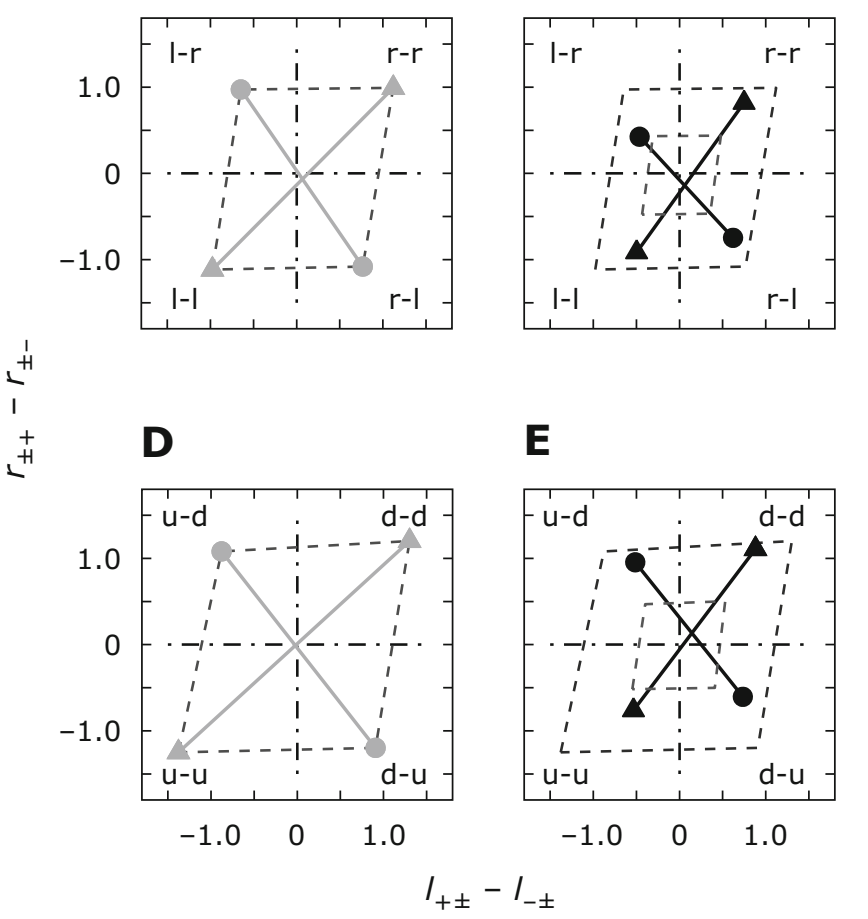

B

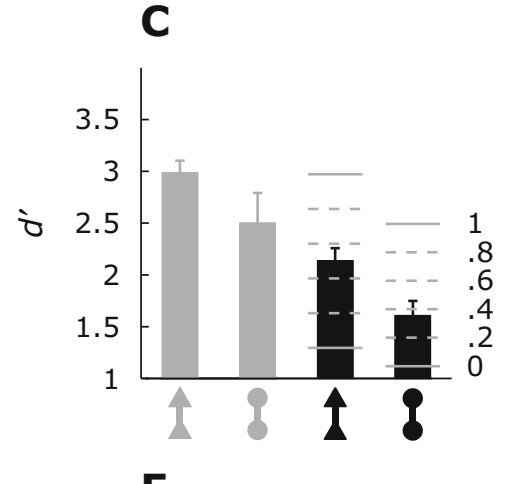

$\mathbf{F}$

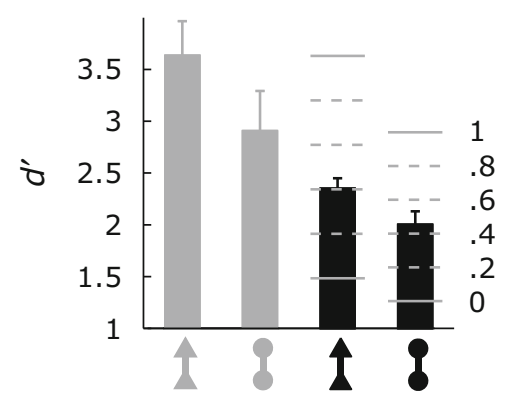

$\cap$<smiles>[18OH]</smiles>

Figure 7. Results for two translational motions (cf. Figure 4). Results for discriminating two horizontal motions $(A, B, C)$ and for discriminating two vertical motions (D, E, F). Single-task (A, D) and dual-task performance (B, E) and comparison of discriminability $d^{\prime}$ and compatibility index $C(C, F)$. In all subfigures, single-task discriminability is marked by gray lines, bars, and symbols (triangles and circles), and dual-task discriminability by black lines, bars, and symbols. The dashed lines in $\mathbf{B}$ and $\mathbf{E}$ mark the maximal and minimal expectations for dual-task performance. The inset scales in $C$ and $F$ mark the compatibility index $C$, which ranges from 0 to $1 . \mathbf{r}$, l, d, and u denote rightward, leftward, downward, and upward motion, respectively. 
motion fields were $M_{\text {cont }}=1.00$ and .71 , respectively. Similarly, because opposite horizontal motion fields partially conform to a continuous radial flow with a center at fixation, the continuity indices for identical and opposite horizontal motion fields were $M_{\text {cont }}=1.00$ and .39 , respectively.

Even under single-task conditions (when one field could be ignored), identical motion combinations were more discriminable than opposite motion combinations (in Figures 7A and 7D, the distance between triangles is larger than the distance between circles). The difference was significant $[t(4)=11.53, p=.001]$ for vertical translations and might have resulted from signal summation.

The dual-task cost was measured by comparing the discriminability of complementary combinations of motion fields (i.e., $u-u$ vs. $d-d$ and $u-d$ vs. $d-u$ for vertical motion; $1-1$ vs. $\mathrm{r}-\mathrm{r}$ and $1-\mathrm{r}$ vs. $\mathrm{r}-\mathrm{l}$ for horizontal motion; see the Method section, Equation 5). The comparison of the observed single-task (Figures 7A and 7D) and dual-task (Figures 7B and 7E) discriminabilities was summarized in terms of a compatibility index $C$ (Figures $7 \mathrm{C}$ and $7 \mathrm{~F}$ ), which rates dual-task discriminability against the maximal and minimal expectations (dashed lines in Figures 7B and 7E; see the Method section for details). A value near unity means that dual-task performance is near maximal; a value near zero implies that dual-task performance is close to minimal.
For horizontal translations, the compatibility of identical motions (1-1 vs. $\mathrm{r}-\mathrm{r})$ was only slightly higher $(M=$ $.50, S D=.14)$ than the compatibility of opposite motions $(1-r$ vs. $r-1)(M=.35, S D=.20$; Figure $7 \mathrm{C})$. For vertical translations, the compatibility of identical motions $(\mathrm{u}-\mathrm{u}$ vs. d-d) was slightly lower than that of opposite motions $(M=.41, S D=.10$, and $M=.46, S D=.16$, respectively; Figure 7F). Neither difference reached significance $(p>.1)$. All figures illustrate the average and standard error over 4 observers. Note that although the discriminability $d^{\prime}$ of identical translation is higher than that of opposite translation, the differences in measures of compatibility are not significant.

Overall, having to divide attention between the left and right fields reduced performance between $50 \%$ and $65 \%$ (as measured by discriminability $d^{\prime}$ ). This result conflicts with an earlier study (Sàenz et al., 2003), which reported lower performance costs when attention was divided between identical motions (see below).

Two circular or radial motion fields. Using either circular or radial motions in both fields, we again investigated displays with identical and with opposite motion in the left and right fields. The identical combinations were $\mathrm{cw}-\mathrm{cw}, \mathrm{cc}-\mathrm{cc}$, out-out, and in-in, and the opposite combinations were $\mathrm{cw}-\mathrm{cc}, \mathrm{cc}-\mathrm{cw}$, in-out, and out-in. By design, identical and opposite motions contrasted starkly in terms of similarity ( $M_{\text {sim }}=1$ and 0 , respectively). In terms of continuity, however, opposite motions rated more
A

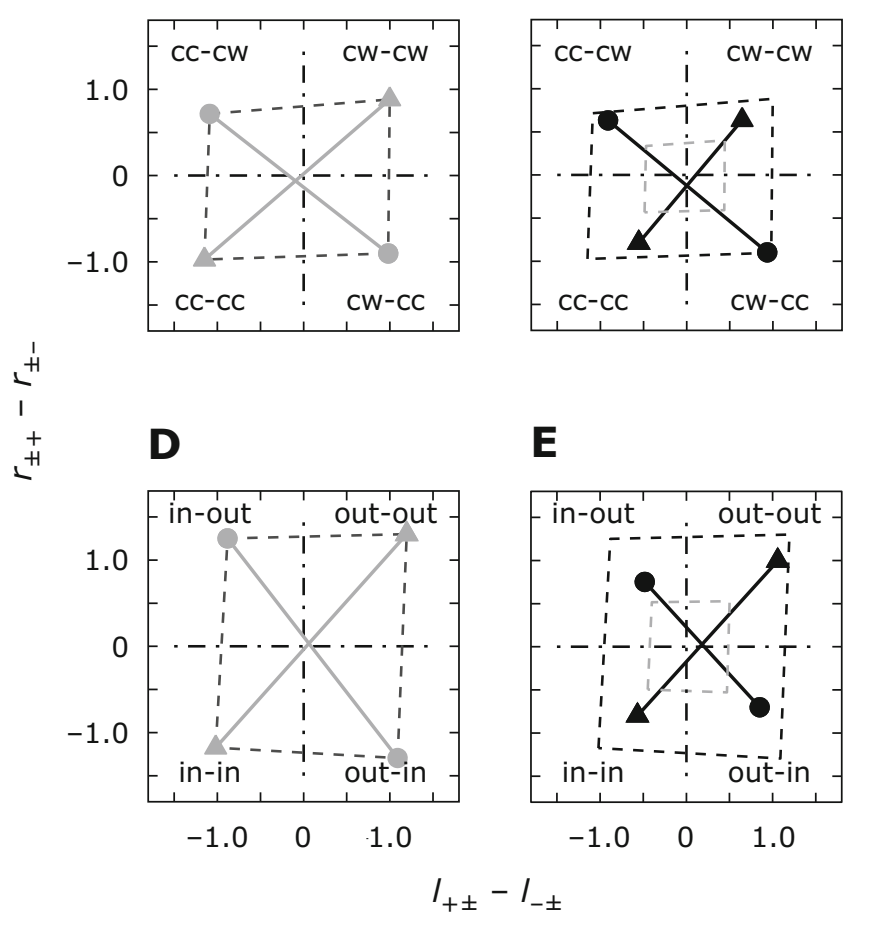

B

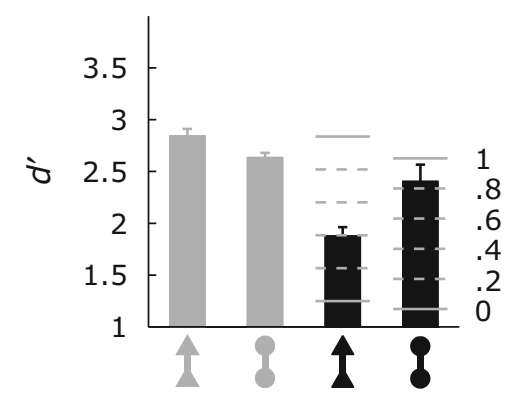

$\mathbf{F}$

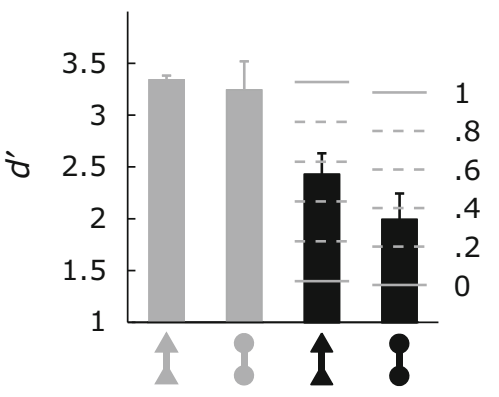

ก

ก

Figure 8. Results for two circular or two radial motions (cf. Figure 4). Results for discriminating two circular motions $(\mathrm{A}, \mathrm{B}, \mathrm{C})$ and for discriminating two radial motions (D, E, F). Single-task (A, D) and dual-task (B, E) performance and comparison of discriminability $d^{\prime}$ and compatibility index $C(C, F)$. In all subfigures, single-task discriminability is marked by gray lines, bars, and symbols (triangles and circles), dual-task discriminability by black lines, bars, and symbols. cw, cc, in, and out denote clockwise, counterclockwise, inward, and outward motion, respectively. 
highly than identical motions $\left(M_{\text {cont }}=.63\right.$ and .20 , respectively, for circular fields, and $M_{\text {cont }}=.15$ and .04 , respectively, for radial fields). This was due to the fact that opposite circular and radial motions partially conform to a continuous translational flow, especially near the center of the display, on which the calculation of our continuity indices placed particular emphasis (see the Method section).

Under single-task conditions, the discriminability of identical combinations was slightly higher than that of opposite combinations (Figures $8 \mathrm{~A}$ and $8 \mathrm{D}$ ). The difference was barely significant $[t(4)=2.858, p=.065]$ for circular motions and was not significant $(p>.1)$ for radial motions.

The dual-task results for circular motion were surprising, in that the compatibility of identical combinations was significantly lower than that of opposite combinations $(M=.40, S D=.10$, and $M=.85, S D=.22$, respectively $)$ $[t(4)=6.65, p=.011$; Figures $8 \mathrm{~B}$ and $8 \mathrm{C}]$. For radial motion, the compatibility of identical combinations was slightly higher than that of opposite combinations $(M=$ $.54, S D=.22$, and $M=.34, S D=.28$, respectively; Figures $8 \mathrm{E}$ and $8 \mathrm{~F}$ ). This difference did not reach significance, however $(p>.1)$.

Overall, having to divide attention caused a performance drop of $45 \%$ to $65 \%$, except in the case of identical circular motions, where performance dropped merely by $15 \%$.
Dissimilar motion fields with little continuity. Next, we investigated dual-task interference when different flow patterns were presented in the left and right motion fields. We combined vertical motion on the left with horizontal motion on the right $(\mathrm{u}-\mathrm{r}, \mathrm{u}-\mathrm{l}, \mathrm{d}-\mathrm{r}$, or $\mathrm{d}-\mathrm{l})$ and radial motion on the left with circular motion on the right (in-cw, out-cw, in-cc, or out-cc). These combinations of motion fields rated comparatively low, both in terms of similarity $\left(M_{\text {sim }}=0\right.$ and .08 , respectively) and of continuity $\left(M_{\text {cont }}=.08\right.$ and .18$)$, since no combination conformed even partially to a continuous flow.

For none of these displays did single-task performance depend significantly on motion in the ignored field (Figures 9A and 9D). The dual-task cost was consistently high. For combinations of vertical and horizontal motions, the compatibility indices of complementary pairs were low $(M=.12, S D=.14$, and $M=.19, S D=.22$; Figures 9B and $9 \mathrm{C})$. For combinations of radial and circular motions, the compatibility indices were low as well $(M=.14, S D=$ .22 , and $M=.02, S D=.08$; Figures $9 \mathrm{E}$ and $9 \mathrm{~F})$. It seems that, in the absence of any significant degree of continuity between left and right motion fields, it is essentially impossible to divide attention.

Dissimilar motion fields with some continuity. Finally, we investigated flow patterns in the left and right motion fields that were dissimilar, but that nevertheless conformed partly to a continuous flow. Specifically, we
A

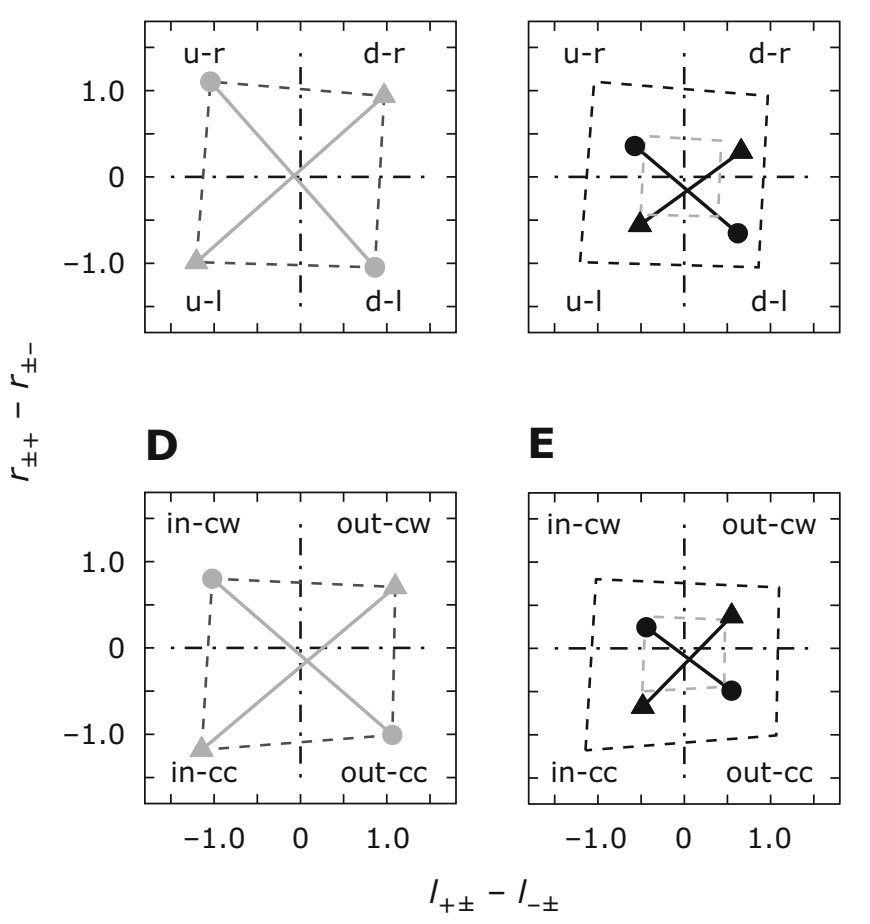

B
Figure 9. Results for dissimilar motions without continuity (cf. Figure 4). Results for discriminating vertical and horizontal motions $(A, B, C)$ and for discriminating radial and circular motions (D, E, F). Single-task (A, D) and dual-task $(B, E)$ performance and comparison of discriminability $d^{\prime}$ and compatibility index $C(C, F)$. In all subfigures, single-task discriminability is marked by gray lines, bars, and symbols (triangles and circles), and dual-task discriminability by black lines, bars, and symbols. r, l, u, d, cw, cc, in, and out stand for rightward, leftward, upward, downward, clockwise, counterclockwise, inward, and outward motion, respectively. 
combined circular motion on the left with vertical motion on the right $(\mathrm{cw}-\mathrm{u}, \mathrm{cw}-\mathrm{d}, \mathrm{cc}-\mathrm{u}$, or $\mathrm{cc}-\mathrm{d})$. Although rating uniformly low in terms of similarity $\left(M_{\text {sim }}=.25\right)$, these combinations of motion fields differed widely in terms of continuity indices, which ranged from $M_{\text {cont }}=$ .43 to $M_{\text {cont }}=.97$. This difference was due to the fact that the more central dots in the combinations $\mathrm{cw}-\mathrm{d}$ and $\mathrm{cc}-\mathrm{u}$ moved in the same direction (down and up, respectively), whereas those in the combinations $\mathrm{cw}-\mathrm{u}$ and $\mathrm{cc}-\mathrm{d}$ moved in opposite directions.

In addition, we combined radial motion on the left with horizontal motion on the right (in-r, in- -1 , out - r, or out -1 , $M_{\text {sim }}=.23$ ). In case of the combinations in -1 and out-r, the more central dots of both fields moved in the same direction $\left(M_{\text {cont }}=.53\right)$, whereas in the case of the combinations in $-\mathrm{r}$ and out -1 , the more central dots moved in opposite directions $\left(M_{\text {cont }}=.01\right)$.

For these displays, single-task performance did not depend significantly on motion in the ignored field (Figures $10 \mathrm{~A}$ and $10 \mathrm{D}$ ). In contrast, dual-task costs depended strongly on the particular combination of motion fields. For combinations of circular and vertical motions, the compatibility index of complementary pairs with some continuity $(\mathrm{cw}-\mathrm{d}$ and $\mathrm{cc}-\mathrm{u}$ ) was significantly higher than the compatibility index of complementary pairs without continuity (cw- $-\mathrm{u}$ and $\mathrm{cc}-\mathrm{d}$ ) $[M=.84, S D=.14$, and $M=.14$, $S D=.12$, respectively; $t(4)=7.02, p=.006$; Figures 10B and $10 \mathrm{C}]$. For combinations of radial and horizontal motions, the compatibility index of complementary pairs with some continuity (in-1 and out-r) was thus again significantly higher than the compatibility index of complementary pairs without continuity (in-r and out -1$)[M=.50$, $S D=.32$, and $M=-.07, S D=.08$, respectively; $t(4)=$ $3.02, p=.05$; Figures $10 \mathrm{E}$ and $10 \mathrm{~F}]$. Accordingly, it would seem that the degree to which two motion fields conform to a continuous flow robustly affects dual-task costs.

\section{Quantitative Analysis}

The results just described suggest several qualitative conclusions. First, whether the more central dots in the left and right fields exhibit common motion (and thus conform to a continuous optic flow) is often, but not always, an important determinant of dual-task costs. With such common motion, such costs are minimal or moderate; without it, they often (but not always) approach the theoretical maximum. Second, even without common motion, one sometimes observes moderate dual-task costs. This is true, for example, when opposite motion flows (either vertical or horizontal) are present on the left and right of fixation, conforming partially to a continuous circular or radial optic flow. Third, the mere similarity of left and right motion flows does not ensure low dual-task costs. For example, identical circular or radial motion flows to the left and right of fixation entail substantial dual-task costs.
A

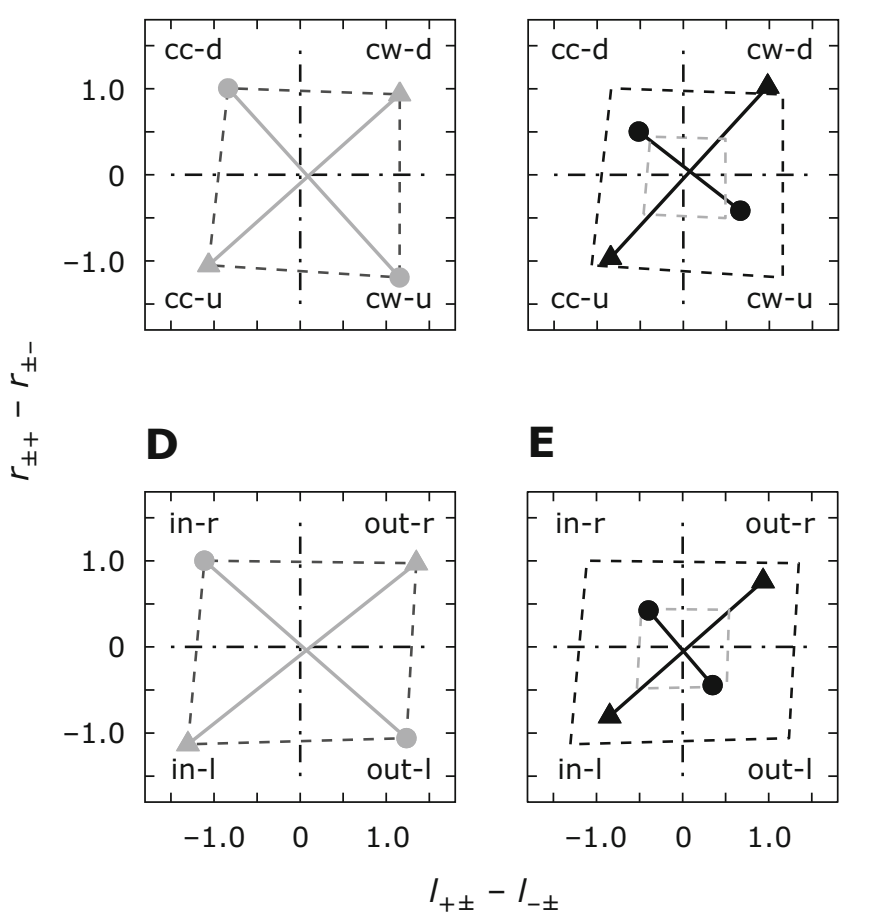

B

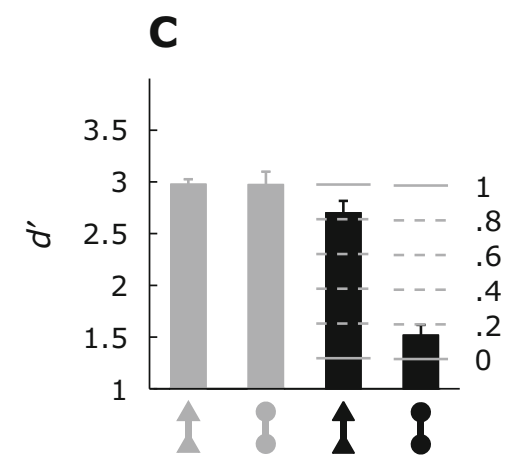

F

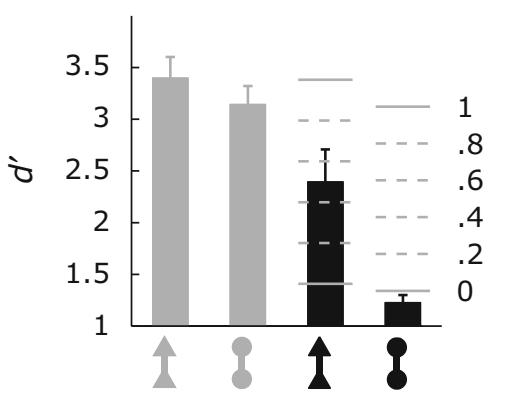

Figure 10. Results for dissimilar motions with some continuity (cf. Figure 4). Results for discriminating circular and vertical motions $(A, B, C)$ and for discriminating radial and horizontal motions (D, E, F). Single-task (A, D) and dual-task $(B, E)$ performance and comparison of discriminability $d^{\prime}$ and compatibility index $C(C, F)$. In all subfigures, single-task discriminability is marked by gray lines, bars, and symbols (triangles and circles), and dual-task discriminability by black lines, bars, and symbols. r, l, u, d, cw, cc, in, and out stand for rightward, leftward, upward, downward, clockwise, counterclockwise, inward, and outward motion, respectively. 


\section{A}

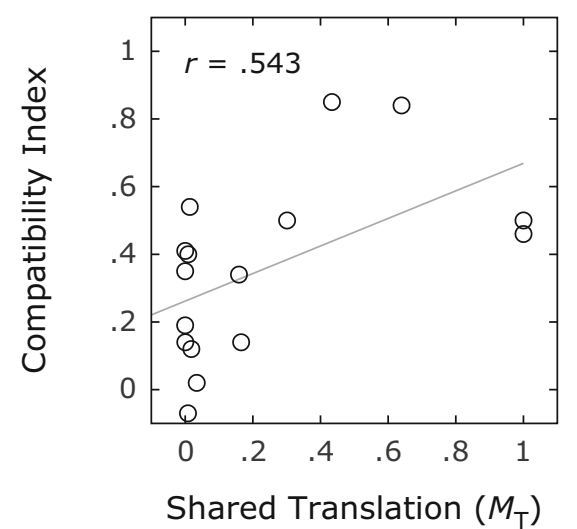

B

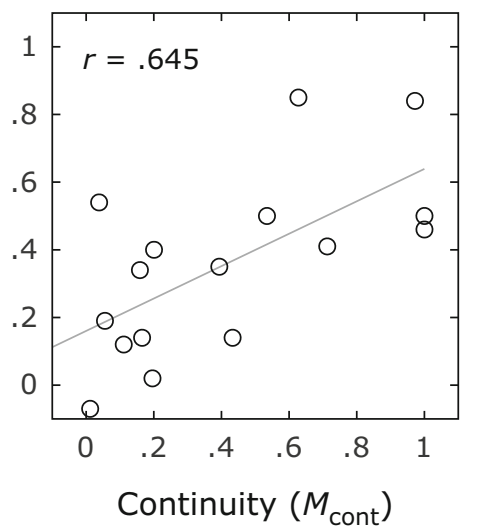

\section{C}

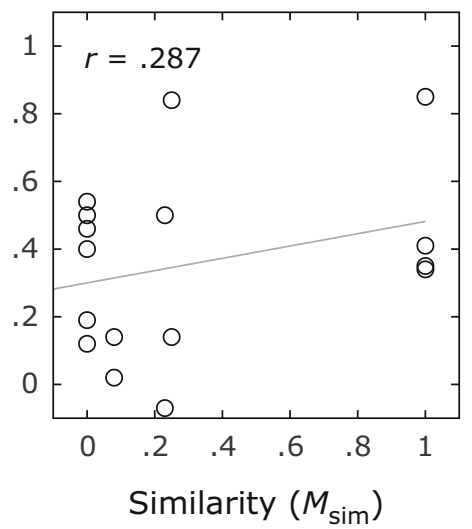

Figure 11. Correlation of compatibility index $C$ with quantitative measures of motion continuity and motion similarity. (A) Correlation with translational continuity $M_{\mathrm{T}}$. (B) Correlation with continuity index $M_{\text {cont }}$ (C) Correlation with similarity index $M_{\text {sim }}$.

To place these conclusions on a more quantitative basis, we devised computational measures for both the similarity and continuity of left and right motion fields (see the Method section for details).

The similarity index $M_{\text {sim }}$ measured the extent to which corresponding portions in the left and right fields exhibited similar patterns of motion. It was computed as the average similarity between motion directions at corresponding locations. High similarity resulted in values near unity, and low similarity resulted in values near zero.

The continuity index $M_{\text {cont }}$ measured the extent to which the left and right motion fields conformed to a continuous optic flow. Taking a Gibsonian point of view, we allowed all ecological types of optic flows, including translational, circular, and radial flows. Accordingly, we first computed how well two motion fields conformed to a particular type of continuous flow-either translational, circular, or radial - denoting the corresponding indices $M_{\mathrm{T}}, M_{\mathrm{C}}$, and $M_{\mathrm{R}}$, respectively. In doing so, we emphasized the innermost portions of the left and right fieldsin other words, the portions in closest proximity to each other. Good conformity to a continuous flow resulted in values near unity, and poor conformity resulted in values near zero. Finally, we computed the value of $M_{\text {cont }}$ as the maximum of the three values $M_{\mathrm{T}}, M_{\mathrm{C}}$, and $M_{\mathrm{R}}$. Thus, $M_{\text {cont }}$ measured how well two motion fields conform to the bestmatching continuous flow, whether this be a translational, circular, or radial flow.

Equipped with these quantitative indices $-M_{\text {cont }}, M_{\mathrm{T}}$, $M_{\mathrm{C}}, M_{\mathrm{R}}, M_{\mathrm{sim}}$-we tried to define more accurately the factors that control the dual-task performance cost of dividing attention between left and right motion fields. To this end, we computed correlation coefficients (Pearson's $r$ ) between various indices of continuity and similarity, on the one hand, and the dual-task compatibility index $C$, on the other hand.

The direct correlation between translational continuity $M_{\mathrm{T}}$ and compatibility $C$ was moderate (Pearson's $r=$ .543 ) but significant (KS test, $p=.028$; Figure 11A). In contrast, the direct correlations between circular or radial continuity indices $M_{\mathrm{C}}$ or $M_{\mathrm{R}}$ and compatibility $C$ were not significant (not shown). However, when the correlation between $M_{\mathrm{T}}$ and $C$ was first subtracted, a linear combination of $M_{\mathrm{R}}$ and $M_{\mathrm{C}}$ correlated with the residual variance of $C$ (Pearson's $r=.461$, KS test, $p=.211$ ), though not significantly. The highest correlation was observed between a linear combination of $M_{\mathrm{T}}, M_{\mathrm{R}}$, and $M_{\mathrm{C}}$ and compatibility $C$ (Pearson's $r=.696, \mathrm{KS}$ test, $p=.041$ ). Multiple regression attributed relative weights of $M_{\mathrm{T}}=.24$, $M_{\mathrm{R}}=.45$, and $M_{\mathrm{C}}=.47$. The direct correlation between the combined continuity index $M_{\text {cont }}$ and compatibility $C$ was significant as well (Pearson's $r=.645, \mathrm{KS}$ test, $p=.007$; Figure 11B). In contrast, the direct correlation between similarity index $M_{\text {sim }}$ and compatibility $C$ was not significant (Pearson's $r=.287, \mathrm{KS}$ test, $p=.281$; Figure 11C). Even when the correlation between $M_{\text {cont }}$ and compatibility $C$ was first subtracted, the similarity index $M_{\text {sim }}$ failed to show a significant correlation with the residual variance of $C$ (Pearson's $r=.269, \mathrm{KS}$ test, $p=.315$ ).

In conclusion, we found no evidence that the similarity of motion patterns systematically reduces dual-task performance costs, as would have been expected from feature-based mechanisms of attentional selection. In contrast, conformance to a continuous flow did systematically and significantly lower dual-task costs, presumably because such conformance facilitates the joint selection of both motion fields by object-based attentional mechanisms. Interestingly, this was the case for different types of continuous flows. For some combinations of motion fields, the most central portions exhibited the same direction of motion and therefore conformed partially to either a translational flow (in-out, out-in, $\mathrm{cw}-\mathrm{cc}, \mathrm{cc}-\mathrm{cw}$ ) or an asymmetric circular or radial flow $(\mathrm{cc}-\mathrm{u}, \mathrm{cw}-\mathrm{d}$, in -1 , out-r). For other combinations, the most central portions of the motion fields exhibited opposite directions of motion and thus conformed partially to a symmetric rotational or translational flow $(\mathrm{u}-\mathrm{d}, \mathrm{d}-\mathrm{u}, \mathrm{l}-\mathrm{r}, \mathrm{r}-\mathrm{l})$. In either case, dual-task performance costs were lower than in control situations in which the two motion fields failed 
to conform to any continuous flow $(\mathrm{cw}-\mathrm{cw}, \mathrm{cc}-\mathrm{cc}$, in-in, out-out, cw-u, cc-d, in-r, out-l, u-r, u-l, d-r, d-l, in-cw, out-cw, in-cc, or out-cc). However, there was one major exception to this pattern of results: Dual-task performance costs were comparatively high when the two motion fields exhibited the same translational motion $(\mathrm{u}-\mathrm{u}, \mathrm{d}-\mathrm{d}, \mathrm{l}-\mathrm{l}$, $r-r)$, even though these combinations conformed perfectly to a continuous translational flow.

\section{DISCUSSION}

In the present study, we compared the respective perceptual benefits of attentional selection for objects and for features. Observers were asked to concurrently and independently discriminate the movement direction of two random-dot fields. In this situation, behavioral performance reflects the extent to which observers are able to attend both fields simultaneously. If both fields can be fully attended during each trial, concurrent performance will be comparable to performance under single-task conditions, in which case we may speak of a low cost (or of a high compatibility). If only one field can be attended during each trial, performance falls midway between single task and chance levels; in this case, the outcome may be described as high cost (or low compatibility).

\section{Object Attention}

Generally speaking, we found that reporting the movement directions of both random-dot fields was highly compatible when these fields conformed to a continuous optic flow. For example, we observed high compatibility with stimulus configurations that were consistent with an overall rotation encompassing both fields (e.g., a clockwise rotation in the left field and a downward translation in the right field). Similarly, we obtained high compatibilities with configurations that were consistent with an overall expansion or contraction (e.g., a rightward translation in the left field and a contraction in the right field). Finally, we observed high compatibility with opposite rotations in left and right fields, which are consistent with a common (upward or downward) translation in the central portion of the display.

Generally speaking again, we observed low compatibility with movement fields that failed to conform to a continuous flow pattern. For example, this was the case for combinations involving two orthogonal translations, for combinations of circular (rotation) and radial motions (expansion/contraction), and for combinations of mismatched circular/radial and translational motions.

These results suggest that, when two local motions can be integrated into one perceptual unit, they may be attended concurrently. Accordingly, these results are consistent with an object-based nature of attentional selection (Driver \& Frith, 2000; Duncan, 1996; Scholl, 2001; Treisman \& Kanwisher, 1998). Furthermore, they corroborate a number of prior reports on the spatial integration of motion features, which have demonstrated that detection and discrimination of local motion are facilitated when the local motion is embedded in a global optic flow (Bravo, 1998; Freeman \& Harris, 1992; Morrone, Burr, \& Vaina, 1995).
However, we also observed a striking exception to this pattern, which is not easily interpretable in terms of object attention. Specifically, we failed to find high compatibility for identical translations in left and right motion fields, even though these combinations conformed perfectly to a continuous translational flow. It is not clear why these combinations should entail higher dual-task performance costs than other combinations that conform only partially to a continuous flow (e.g., two opposing circular motions). We speculate that whole-field translational optical flows may be too frequently induced by self-motion to count as sufficient evidence for the presence of a common visual object. If so, then the presence of additional optic flows in other directions, which would rule out self-motion, might change the situation. Thus, dual-task performance costs for two identical translations might decrease for more complex displays that also contain additional optic flows. Indeed, Sàenz et al. (2003) reported reduced dual-task interference for more complex displays, in which each motion field contained two sets of dots moving in opposite directions. This study is discussed in more detail below.

Several previous studies have manipulated the ease or difficulty with which different stimulus components are integrated into a single perceptual unit. All have reported similar effects on dual-task compatibility. Watson and Kramer (1999) asked observers to report on the terminal shapes of an elongated outline. Joint segmentation of the terminal shapes was promoted or hindered by introducing parsing cues such as concavities, discontinuities, and so forth, into the outline. When joint segmentation was hindered by strong parsing cues, the difficulty of reporting on two terminals of one outline was comparable to that of reporting on terminals of two different outlines. Barenholtz and Feldman (2003) used a "peanut"-shaped object with four distinct lobes. Once again, parsing cues (outline curvature) hindered attentional selection: the stronger such cues, the longer the latency of reports regarding different lobes. Most similar in spirit to our study is the work of BenShahar, Scholl, and Zucker (2007), who directly linked attentional selection to scene segmentation. Two letter shapes were superimposed over a background texture of oriented elements that was either continuous or discontinuous. Report accuracy was higher when the shapes appeared within a continuous as compared with a discontinuous texture.

\section{Feature Attention}

None of our results conformed to the expectations of a hypothetical feature-based selection. To wit, we never observed higher compatibility with stimulus configurations combining similar types of motion flow than with configurations combining dissimilar motion flows. For example, a significantly higher compatibility was evident neither when comparing two identical with two opposite rotations, nor when comparing two identical radial motions (e.g., two contractions) with two opposite radial motions (e.g., a contraction and an expansion). Similarly, no significantly higher compatibility was apparent for parallel motions (e.g., two rightward translations) than for opposite motions (e.g., one rightward and one leftward translation). A computational analysis of our results revealed 
no evidence of even a residual contribution of similarity to dual-task compatibility.

From one point of view, this negative result is unsurprising and follows ample precedent. Early comparisons of the dual-task compatibilities of similar and dissimilar pairs of discriminations consistently found comparable degrees of conflict/compatibility (Duncan, 1993; Duncan \& Nimmo-Smith, 1996; Ward, Duncan, \& Shapiro, 1997). More recent studies, in which the performance trade-off between pairs of discriminations was fully established (i.e., the attention-operating characteristic traced) demonstrated identical degrees of conflict/compatibility between similar and dissimilar discriminations (Lee et al., 1999a, 1999b; Pastukhov et al., 2009). One should emphasize, however, that these studies contrasted the similarity or dissimilarity of feature dimensions, not of feature values. In other words, the similar task combinations involved, for example, two color discriminations, or two motion discriminations, whereas the dissimilar task combinations involved one color and one motion discrimination. In the present study, similarity and dissimilarity referred to the feature values (i.e., types of motion flow) within the same feature dimension.

The best evidence that feature attention may benefit perceptual performance comes from visual search paradigms. To the extent that feature attention contributes to search performance, it appears equally capable of selecting a feature dimension or a feature value. The improvements in search performance that spring from knowing the target dimension or the target feature have been documented by several studies (Müller et al., 1995; Treisman, 1988; Wolfe, Butcher, Lee, \& Hyle, 2003). Typically, a cross-dimensional search in which the target may be identified by any one of several dimensions (e.g., color, motion) is faster than an open search, but slower than a within-dimension search, in which target values (e.g., red, green, blue) vary within a single dimension (e.g., color). The dimension-specific facilitation is typically attributed to attentional signals that assign greater weight to the target dimension and thus allow faster target detection (Müller et al., 1995; Müller, Reimann, \& Krummenacher, 2003). Note, however, that the benefit of feature guidance increases when the target features remain the same from trial to trial (Wolfe et al., 2004; Wolfe, Horowitz, Palmer, Michod, \& Van Wert, 2010), so that a selectional bias can build up gradually ("consistent mapping"; Schneider \& Shiffrin, 1977; Shiffrin \& Schneider, 1977).

The results of the single previous dual-task study of feature-based attention were consistent with ours. Sàenz et al. (2003) presented two fields of dots, one on either side of fixation, that either exhibited the same feature (same direction of motion, 100\% coherence) or different features (opposite directions of motion, $100 \%$ coherence), and found identical dual-task performance in both situations (as we did). The outcome changed only when each motion field contained two sets of dots moving in opposite directions (50\% coherence each) - in other words, when the two competing features where transparently overlaid. In this situation, it did indeed prove easier to report on two parallel than on two opposite motions. Interestingly, this experiment was conducted under "consistent mapping" conditions in that observers attended the same features on successive trials (e.g., up on the left, down on the right).

Sàenz et al. (2003) argued that the transparent overlay, which forced observers to filter out competing stimuli at the same location, engaged attention more strongly than did the situation without overlay. This would be consistent with neurophysiological findings that attentional effects on single-cell responses are greater when both task-relevant and task-irrelevant stimuli are present in the receptive field (Luck, Chelazzi, Hillyard, \& Desimone, 1997; Moran \& Desimone, 1985; Motter, 1993; Treue \& Martínez-Trujillo, 1999; Treue \& Maunsell, 1999).

In designing the present study, we were well aware of the importance of an attentionally challenging situation. Unfortunately, the different types of motion flow that we wished to employ (i.e., translation, rotation, expansion/ contraction) proved too complex for a transparent overlay. Even after intensive training, observers were unable to segment a left and a right motion flow from two additional, overlayed motion flows in a dual-task situation. To create a compromise between the two situations studied by Sàenz et al. (2003; single motion flows of $100 \%$ coherence or overlayed motion flows of $50 \%$ coherence each), we used single motion flows of low coherence (average coherence $20 \%$, range $6 \%$ to $37 \%$ ). We expected that the need to filter out $80 \%$ noise dots would be sufficient to strongly engage attention. This expectation was borne out by the strong object-based attention effects summarized above. Accordingly, the lack of feature-based attention effects in our situation cannot be attributed to an oversimple stimulus that failed to engage attention.

\section{Neurophysiological Evidence}

Much of the evidence in favor of feature attention is neurophysiological. In general, feature attention is demonstrated by transparently superimposing in one region of the display two stimulus patterns that are distinguished by a particular feature (e.g., direction of motion, orientation, color). When a trained nonhuman primate attends to one pattern (specifically to its distinguishing feature) and ignores the other pattern, neural responses to stimuli presented in unattended regions of the display are modulated. Typically, responses are enhanced for neurons that are selective to the attended feature and suppressed for neurons selective to the ignored feature (Haenny, Maunsell, \& Schiller, 1988; Maunsell \& Treue, 2006; Motter, 1994a, 1994b; Treue \& Martínez-Trujillo, 1999). Analogous results have been obtained with human observers using fMRI (Sàenz et al., 2002; Serences \& Boynton, 2007) and evoked potential recordings (Müller et al., 2006).

This spreading of an attentional modulation to stimuli well outside the attended region is sufficiently robust to be detected with indirect, behavioral methods. For example, subthreshold priming by an unattended colored stimulus is significantly enhanced when observers attend to a stimulus of the same color in the opposite hemifield (Melcher, Papathomas, \& Vidnyánszky, 2005). Similarly, the motion aftereffect induced by an unattended moving stimulus is enhanced when observers attend to the same 
motion in the opposite hemifield (Boynton, Ciaramitaro, \& Arman, 2006).

Recently, it has been suggested that the modulations attributed to feature attention may actually spread in an object-specific manner. Wannig, Rodríguez, and Freiwald (2007) trained nonhuman primates to attend to one surface of rotating dot pattern within a stimulus that consisted of two sets of differently colored dots rotating in opposite directions and found attentional enhancement of neurons selective also to consistent translational flows. This implies that the enhancement is not restricted to a particular feature (linear motion) but also spreads to dissimilar features (rotational motion) of the same object (rotating surface) (Treue \& Katzner, 2007). These findings raise the possibility that attentional modulations of neurophysiological activity may only appear to be feature-specific and may in actuality reflect object-specific mechanisms of attentional selection.

\section{Conclusion}

We have investigated a number of situations in which object-based attention significantly improves dual-task performance. Specifically, we found that joint selection of two target fields by object-based mechanisms requires a (potentially) continuous motion flow across both fields. In several analogous situations, we found no evidence whatsoever that feature-based attention improves dualtask performance. Specifically, we found that the similarity or dissimilarity of two motion fields made no difference to dual-task performance. All of our results were obtained under conditions of variable mapping (Schneider \& Shiffrin, 1977; Shiffrin \& Schneider, 1977), in that the attended and reported attributes of the two motion fields changed from trial to trial. Note that variable mapping does not impair the effectiveness of object-based mechanisms of selection (e.g., Bauer \& Braun, 2000).

Our negative results regarding feature-based selection contrast with the positive perceptual evidence for feature-based attention that has accumulated over the years, mainly under conditions of consistent mapping. For example, in the one dual-task study that revealed superior performance for two similar (rather than dissimilar) motion fields, the same combination of motion fields was presented for 36 successive trials (Sàenz et al., 2003). In visual search paradigms, the effect of feature guidance increases when a selectional bias can build over successive trials (Müller et al., 1995; Müller et al., 2003; Treisman, 1988; Wolfe et al., 2003; Wolfe et al., 2004; Wolfe et al., 2010).

We conclude that feature-based attentional selection does not seem to operate in the same flexible, short-term, single-trial fashion in which object-based selection does. Rather, feature-based selection seems to build gradually over successive trials. Future experiments on object- and feature-based selection would do well to keep these disparate operating modes in mind.

\section{AUTHOR NOTE}

The present work was supported by the BMBF Bernstein Network for Computational Neuroscience and by the State of Saxony-Anhalt. Cor- respondence concerning this article should be addressed to J. Braun, Institute of Biology, Otto-von-Guericke-Universität, Leipziger Strasse 44 / Haus 91, 39120 Magdeburg, Germany (e-mail: jochen.braun@ovgu.de).

\section{REFERENCES}

Andersen, S. K., Hillyard, S. A., \& Müller, M. M. (2008). Attention facilitates multiple features in parallel in human visual cortex. Current Biology, 18, 1006-1009.

Barenholtz, E., \& Feldman, J. (2003). Visual comparisons within and between object parts: Evidence for a single-part superiority effect. Vision Research, 43, 1655-1666.

BAUER, H. U., \& Braun, J. (2000). Is there parallel binding of distributed objects? In H. Cruse, J. Dean, \& H. Ritter (Eds.), Preparational intelligence: Adaptive behavior and intelligent systems without symbols and logic (Vol. 1, pp. 163-174). Dordrecht, The Netherlands: Kluwer.

Ben-Shahar, O., Scholl, B. J., \& Zucker, S. W. (2007). Attention, segregation, and textons: Bridging the gap between object-based attention and texton-based segregation. Vision Research, 47, 845-860.

Blaser, E., Pylyshyn, Z. W., \& Holcombe, A. O. (2000). Tracking an object through feature space. Nature, 408, 196-199.

Boynton, G. M., Ciaramitaro, V. M., \& Arman, A. C. (2006). Effects of feature-based attention on the motion aftereffect at remote locations. Vision Research, 46, 2968-2976.

Brainard, D. H. (1997). The Psychophysics Toolbox. Spatial Vision, 10, 433-436.

Bravo, M. J. (1998). A global process in motion segregation. Vision Research, 38, 853-863.

Driver, J., \& Frith, C. (2000). Shifting baselines in attention research. Nature Reviews Neuroscience, 1, 147-148.

Duncan, J. (1984). Selective attention and the organization of visual information. Journal of Experimental Psychology: General, 113, 501517.

DUNCAN, J. (1993). Similarity between concurrent visual discriminations: Dimensions and objects. Perception \& Psychophysics, 54, 425430.

DunCAN, J. (1996). Cooperating brain systems in selective perception and action. In T. Inui \& J. L. McClelland (Eds.), Attention and performance XVI: Information integration in perception and communication (pp. 549-578). Cambridge, MA: MIT Press.

Duncan, J., \& Nimmo-Smith, I. (1996). Objects and attributes in divided attention: Surface and boundary systems. Perception \& Psychophysics, 58, 1076-1084.

Freeman, T. C., \& Harris, M. G. (1992). Human sensitivity to expanding and rotating motion: Effects of complementary masking and directional structure. Vision Research, 32, 81-87.

Haenny, P. E., Maunsell, J. H., \& Schiller, P. H. (1988). State dependent activity in monkey visual cortex: II. Retinal and extraretinal factors in V4. Experimental Brain Research, 69, 245-259.

LeE, D. K., Koch, C., \& Braun, J. (1999a). Attentional capacity is undifferentiated: Concurrent discrimination of form, color, and motion. Perception \& Psychophysics, 61, 1241-1255.

LEE, D. K., Koch, C., \& Braun, J. (1999b). Visual attention is undifferentiated also for less demanding tasks. Investigative Ophthalmology \& Visual Science, 40(Suppl.), 52.

LiU, T., LARsson, J., \& CARrasco, M. (2007). Feature-based attention modulates orientation-selective responses in human visual cortex. Neuron, 55, 313-323.

Luck, S. J., Chelazzi, L., Hillyard, S. A., \& Desimone, R. (1997). Neural mechanisms of spatial selective attention in areas V1, V2, and V4 of macaque visual cortex. Journal of Neurophysiology, 77, 24-42.

Martínez-Trujillo, J. C., \& Treue, S. (2004). Feature-based attention increases the selectivity of population responses in primate visual cortex. Current Biology, 14, 744-751.

Maunsell, J. H., \& Treue, S. (2006). Feature-based attention in visual cortex. Trends in Neurosciences, 29, 317-322.

Melcher, D., Papathomas, T. V., \& Vidnyánszky, Z. (2005). Implicit attentional selection of bound visual features. Neuron, 46, 723-729.

Moran, J., \& Desimone, R. (1985). Selective attention gates visual processing in the extrastriate cortex. Science, 229, 782-784.

Morrone, M. C., Burr, D. C., \& VAinA, L. M. (1995). Two stages of visual processing for radial and circular motion. Nature, 376, 507-509. 
Motter, B. C. (1993). Focal attention produces spatially selective processing in visual cortical areas V1, V2, and V4 in the presence of competing stimuli. Journal of Neurophysiology, 70, 909-919.

MotTER, B. C. (1994a). Neural correlates of attentive selection for color or luminance in extrastriate area V4. Journal of Neuroscience, 14, 2178-2189.

MotTer, B. C. (1994b). Neural correlates of feature selective memory and pop-out in extrastriate area V4. Journal of Neuroscience, 14, 2190-2199.

Müller, M. M., Andersen, S., Trujillo, N. J., Valdés-Sosa, P., Malinowski, P., \& Hillyard, S. A. (2006). Feature-selective attention enhances color signals in early visual areas of the human brain. Proceedings of the National Academy of Sciences, 103, 14250-14254.

Müller, H. J., Heller, D., \& Ziegler, J. (1995). Visual search for singleton feature targets within and across feature dimensions. Perception \& Psychophysics, 57, 1-17.

Müller, H. J., Reimann, B., \& Krummenacher, J. (2003). Visual search for singleton feature targets across dimensions: Stimulusand expectancy-driven effects in dimensional weighting. Journal of Experimental Psychology: Human Perception \& Performance, 29, 1021-1035.

Norman, D. A., \& Bobrow, D. G. (1975). On data-limited and resourcelimited processes. Cognitive Psychology, 7, 44-64.

O'Craven, K., Downing, P. E., \& Kanwisher, N. (1999). fMRI evidence for objects as the units of attentional selection. Nature, 401, 584-587.

Pashler, H. E. (1999). The psychology of attention. Cambridge, MA: MIT Press.

Pastukhov, A., Fischer, L., \& Braun, J. (2009). Visual attention is a single, integrated resource. Vision Research, 49, 1166-1173.

Pelli, D. G. (1997). The VideoToolbox software for visual psychophysics: Transforming numbers into movies. Spatial Vision, 10, 437-442.

Rodríguez, V., Valdés-Sosa, M., \& Freiwald, W. (2002). Dividing attention between form and motion during transparent surface perception. Cognitive Brain Research, 13, 187-193.

Roelfsema, P. R., Lamme, V. A. F., \& Spekreisse, H. (1998). Objectbased attention in the primary visual cortex of the macaque monkey. Nature, 395, 376-381

SÀEnz, M., Buracas, G. T., \& Boynton, G. M. (2002). Global effects of feature-based attention in human visual cortex. Nature Neuroscience, 5, 631-632.

Sàenz, M., Buracas, G. T., \& Boynton, G. M. (2003). Global featurebased attention for motion and color. Vision Research, 43, 629-637.

SCHNEIDER, W., \& ShIFFrin, R. M. (1977). Controlled and automatic human information processing: I. Detection, search, and attention. Psychological Review, 84, 1-66.

Schoenfeld, M. A., Tempelmann, C., Martinez, A., Hopf, J.-M., Sattler, C., Heinze, H.-J., \& Hillyard, S. A. (2003). Dynamics of feature binding during object selective attention. Proceedings of the National Academy of Sciences, 100, 11806-11811.

Scholl, B. J. (2001). Objects and attention: The state of the art. Cognition, 80, 1-46.

Serences, J. T., \& Boynton, G. M. (2007). Feature-based attentional modulations in the absence of direct visual stimulation. Neuron, $\mathbf{5 5}$, 301-312.
ShifFrin, R. M., \& SCHNeIDER, W. (1977). Controlled and automatic human information processing: II. Perceptual learning, automatic attending, and a general theory. Psychological Review, 84, 127-190.

Sperling, G., \& Dosher, B. (1986). Strategy and optimization in human information processing. In K. R. Boff, L. Kaufman, \& J. P. Thomas (Eds.), Handbook of perception and human performance (Vol. 1, pp. 1-65). New York: Wiley.

Treisman, A. M. (1988). Features and objects: The fourteenth Bartlett Memorial Lecture. Quarterly Journal of Experimental Psychology, 40A, 201-237.

Treisman, A. [M.], \& Kanwisher, N. (1998). Perceiving visuallypresented objects: Recognition, awareness, and modularity. Current Opinion in Neurobiology, 8, 218-226.

Treue, S., \& Katzner, S. (2007). Visual attention: Of features and transparent surfaces. Trends in Cognitive Sciences, 11, 451-453.

Treue, S., \& MartíneZ-Trujillo, J. C. (1999). Feature-based attention influences motion processing gain in macaque visual cortex. Nature, 399, 575-579.

Treue, S., \& Maunsell, J. H. R. (1996). Attentional modulation of visual motion processing in cortical areas MT and MST. Nature, 382, 539-541.

Treue, S., \& Maunsell, J. H. R. (1999). Effects of attention on the processing of motion in macaque middle temporal and medial superior temporal visual cortical areas. Journal of Neuroscience, $\mathbf{1 9}$, 7591-7602.

Valdés-Sosa, M., Bobes, M. A., Rodríguez, V., \& Pinilla, T. (1998) Switching attention without shifting the spotlight: Object-based attentional modulation of brain potentials. Journal of Cognitive Neuroscience, 10, 137-151.

Wannig, A., Rodríguez, V., \& Freiwald, W. A. (2007). Attention to surfaces modulates motion processing in extrastriate area MT. Neuron, 54, 639-651.

WARD, R., Duncan, J., \& Shapiro, K. (1997). Effects of similarity, difficulty, and nontarget presentation on the time course of visual attention. Perception \& Psychophysics, 59, 593-600.

Watson, S. E., \& Kramer, A. F. (1999). Object-based visual selective attention and perceptual organization. Perception \& Psychophysics, 61, 31-49.

WoLfe, J. M. (1994). Guided Search 2.0: A revised model of visual search. Psychonomic Bulletin \& Review, 1, 202-238.

Wolfe, J. M., Butcher, S. J., Lee, C., \& Hyle, M. (2003). Changing your mind: On the contributions of top-down and bottom-up guidance in visual search for feature singletons. Journal of Experimental Psychology: Human Perception \& Performance, 29, 483-502.

Wolfe, J. M., Horowitz, T. S., Kenner, N., Hyle, M., \& Vasan, N. (2004). How fast can you change your mind? The speed of top-down guidance in visual search. Vision Research, 44, 1411-1426.

Wolfe, J. M., Horowitz, T. S., Palmer, E. M., Michod, K. O., \& VaN WERT, M. J. (2010). Getting in to guided search. In V. Coltheart (Ed.), Tutorials in visual cognition (pp. 93-120). Hove, U.K.: Psychology Press.

(Manuscript received July 17, 2009; revision accepted for publication June 8, 2010.) 